\title{
Model Development for Atomic Force Microscope Stage Mechanisms
}

\author{
Ralph C. Smith and Andrew G. Hatch \\ Department of Mathematics \\ Center for Research in Scientific Computation \\ North Carolina State University \\ Raleigh, NC 27695 \\ aghatch@eos.ncsu.edu,rsmith@eos.ncsu.edu \\ Ricardo C.H. del Rosario \\ Department of Mathematics \\ University of the Philippines \\ Diliman, Quezon City 1101 \\ rcdelros@math.upd.edu.ph
}

\author{
Tathagata De and Murti V. Salapaka \\ Electrical Engineering Department \\ Iowa State University \\ Ames, IA 50011 \\ tatha@iastate.edu,murti@iastate.edu
}

\author{
Julie K. Raye \\ Department of Mathematics \\ Virginia Commonwealth University \\ Richmond VA 23284 \\ jkraye@yahoo.com
}

\begin{abstract}
In this paper, we develop nonlinear constitutive equations and resulting system models quantifying the nonlinear and hysteretic field-displacement relations inherent to lead zirconate titanate (PZT) devices employed in atomic force microscope stage mechanisms. We focus specifically on PZT rods utilizing $d_{33}$ motion and PZT shells driven in $d_{31}$ regimes, but the modeling framework is sufficiently general to accommodate a variety of drive geometries. In the first step of the model development, lattice-level energy relations are combined with stochastic homogenization techniques to construct nonlinear constitutive relations which accommodate the hysteresis inherent to ferroelectric compounds. Secondly, these constitutive relations are employed in classical rod and shell relations to construct system models appropriate for presently employed nanopositioner designs. The capability of the models to quantify the frequency-dependent hysteresis inherent to the PZT stages is illustrated through comparison with experimental data.
\end{abstract}




\section{Report Documentation Page}

Form Approved

OMB No. 0704-0188

Public reporting burden for the collection of information is estimated to average 1 hour per response, including the time for reviewing instructions, searching existing data sources, gathering and maintaining the data needed, and completing and reviewing the collection of information. Send comments regarding this burden estimate or any other aspect of this collection of information,

including suggestions for reducing this burden, to Washington Headquarters Services, Directorate for Information Operations and Reports, 1215 Jefferson Davis Highway, Suite 1204, Arlington

VA 22202-4302. Respondents should be aware that notwithstanding any other provision of law, no person shall be subject to a penalty for failing to comply with a collection of information if it

does not display a currently valid OMB control number.

\begin{tabular}{|c|c|c|}
\hline $\begin{array}{l}\text { 1. REPORT DATE } \\
\mathbf{2 0 0 5}\end{array}$ & 2. REPORT TYPE & $\begin{array}{l}\text { 3. DATES COVERED } \\
\text { - }\end{array}$ \\
\hline \multirow{3}{*}{\multicolumn{2}{|c|}{$\begin{array}{l}\text { 4. TITLE AND SUBTITLE } \\
\text { Model Development for Atomic Force Microscope Stage Mechanisms }\end{array}$}} & 5a. CONTRACT NUMBER \\
\hline & & 5b. GRANT NUMBER \\
\hline & & 5c. PROGRAM ELEMENT NUMBER \\
\hline \multirow{3}{*}{\multicolumn{2}{|c|}{ 6. AUTHOR(S) }} & 5d. PROJECT NUMBER \\
\hline & & 5e. TASK NUMBER \\
\hline & & 5f. WORK UNIT NUMBER \\
\hline \multicolumn{2}{|c|}{$\begin{array}{l}\text { 7. PERFORMING ORGANIZATION NAME(S) AND ADDRESS(ES) } \\
\text { Defense Advanced Research Projects Agency,3701 North Fairfax } \\
\text { Drive,Arlington,VA,22203-1714 }\end{array}$} & $\begin{array}{l}\text { 8. PERFORMING ORGANIZATION } \\
\text { REPORT NUMBER }\end{array}$ \\
\hline \multirow{2}{*}{\multicolumn{2}{|c|}{ 9. SPONSORING/MONITORING AGENCY NAME(S) AND ADDRESS(ES) }} & 10. SPONSOR/MONITOR'S ACRONYM(S) \\
\hline & & $\begin{array}{l}\text { 11. SPONSOR/MONITOR'S REPORT } \\
\text { NUMBER(S) }\end{array}$ \\
\hline
\end{tabular}

12. DISTRIBUTION/AVAILABILITY STATEMENT

Approved for public release; distribution unlimited

13. SUPPLEMENTARY NOTES

The original document contains color images.

14. ABSTRACT

see report

15. SUBJECT TERMS

16. SECURITY CLASSIFICATION OF:

\begin{tabular}{c|c|c}
$\begin{array}{c}\text { a. REPORT } \\
\text { unclassified }\end{array}$ & $\begin{array}{c}\text { b. ABSTRACT } \\
\text { unclassified }\end{array}$ & $\begin{array}{c}\text { c. THIS PAGE } \\
\text { unclassified }\end{array}$
\end{tabular}

17. LIMITATION OF
ABSTRACT

\begin{tabular}{l|l}
$\begin{array}{l}\text { 18. NUMBER } \\
\text { OF PAGES } \\
\mathbf{2 8}\end{array}$ & 19a. NAME OF \\
& RESPONSIBLE PERSON \\
& \\
\hline
\end{tabular}




\section{Introduction}

Stage mechanisms employing the ferroelectric material lead zirconate titanate (PZT) have played a fundamental role in scanning tunneling microscope (STM) and atomic force microscope (AFM) design since their inception due to the high set point accuracy, large dynamic range, and relatively small temperature sensitivity exhibited by the compounds [10]. To illustrate, consider the prototypical AFM design depicted in Figure 1. To ascertain the 3-D surface structure of a sample, it is moved laterally along a pre-determined $x-y$ grid by a PZT-driven stage. The response of a highly flexible microcantilever to changing atomic surface forces is monitored by a reflected laser beam measured via a photodiode, and forces corresponding to the cantilever displacement changes are determined via Hooke's law. A feedback law is used to determine voltages to a transverse PZT stage which produces displacements in the $z$-direction to maintain constant forces. A complete scan in this manner provides a surface image of the compounds. Additionally, PZT actuators are often used to drive the microcantilevers at resonance to achieve the tapping mode operation used to reduce damage to specimens.

Two representative stage designs are depicted in Figure 2. The first employs stacked PZT actuators utilizing $d_{33}$ electromechanical motion to achieve longitudinal positioning along the pre-specified $x-y$ grid. A second stage provides the transverse motion required to ascertain the sample topography. Rod models with linear and nonlinear electromechanical input relations are constructed to quantify the PZT transducer dynamics in this design. The second geometry employs a cylindrical shell with half poled $d_{33}$ to provide horizontal $(x-y)$ motion and half poled $d_{31}$ for vertical $(z)$ motion as depicted in Figure 2(b) - to enhance vibration isolation and reduce hysteresis and constitutive nonlinearities. Thin shell models are developed to characterize this stage design.

To illustrate issues which must be addressed by models, field-displacement data from the stacked actuator depicted in Figure 2(a) is plotted in Figures 3 and 4. The data in Figure 3 was collected at $0.1 \mathrm{~Hz}$ and illustrates the nested, hysteretic relation between input fields and generated displacements in a nearly quasistatic regime. The data in Figure 4 was collected at frequencies ranging from $0.279 \mathrm{~Hz}$ to $27.9 \mathrm{~Hz}$ to illustrate the frequency-dependence of the hysteresis as well as certain dynamic effects.

At low frequencies, the inherent hysteresis can be accommodated through proportional-integralderivative (PID) or robust control designs [5, 6, 18, 24]. However, at the higher frequencies required for applications including real-time monitoring of biological processes (e.g., protein unfolding), comprehensive product diagnostics, and single electron spin detection $[23,34]$, increasing noise-to-data

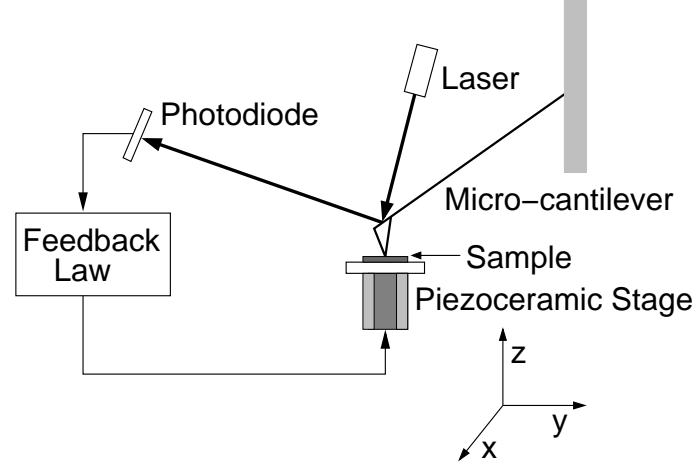

(a)

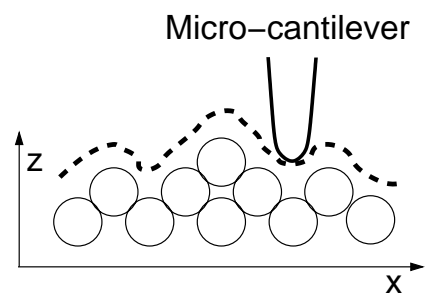

(b)

Figure 1: (a) Configuration of a prototypical AFM, and (b) surface image determined by one lateral sweep. 


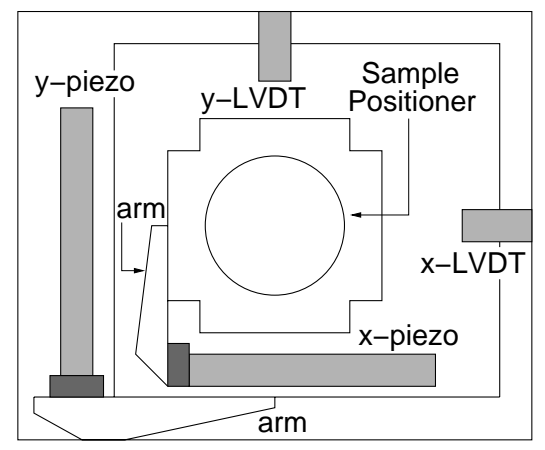

(a)

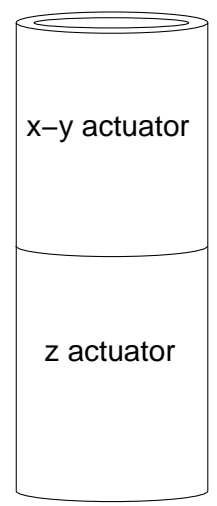

(b)

Figure 2: Actuator configurations employed for sample positioning in AFM: (a) stacked actuators employed as $x$ - and $y$-stages, and (b) cylindrical PZT transducer.

ratios and diminishing high-pass characteristics of control filters preclude a sole reliance on feedback laws to eliminate hysteresis.

Alternatively, it is illustrated in $[16,17]$, that use of charge- or current-controlled amplifiers can essentially eliminate hysteresis. However, this mode of operation can be prohibitively expensive when compared with the more commonly employed voltage-controlled amplifiers, and current control is ineffective if maintaining DC offsets as is the case when the $x$-stage of an AFM is held in a fixed position while a sweep is performed with the $y$-stage.

The need to significantly increase scanning speeds with general amplifiers motivates the development of models and model-based control designs which accommodate the frequency-dependent hysteresis inherent to the PZT actuators employed in the AFM stages. As detailed in [25], there exist a number of general approaches and frameworks for quantifying the constitutive nonlinearities and hysteresis in the general class of ferroelectric materials which encompass PZT. These include phenomenological macroscopic models [20], Preisach models [9, 22], domain wall models [28, 29], micromechanical models $[4,14,15]$, mesoscopic energy relations $[3,13]$ and homogenized energy models $[27,33]$. Within the context of AFM design, Croft, Shed and Devasia [5] have employed a combination of a viscoelastic creep model and nonlinear Preisach representation to compensate for hysteresis and creep in an AFM stage whereas a domain wall model was employed in [30] for the

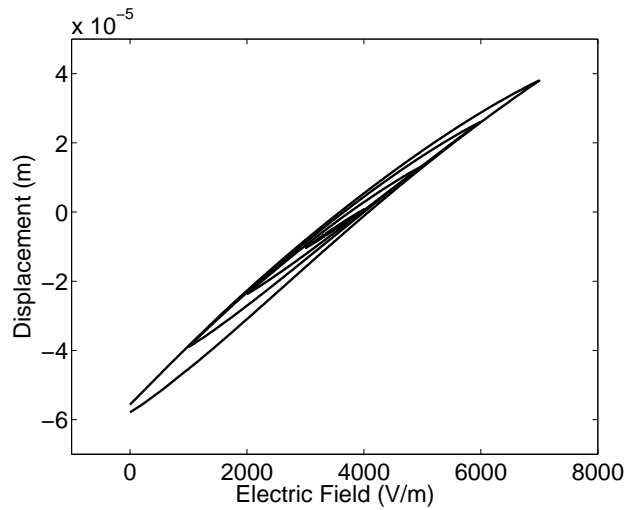

Figure 3: Nested minor loops in $0.1 \mathrm{~Hz}$ field-displacement data from a stacked PZT stage of the type depicted Figure 2(a). 


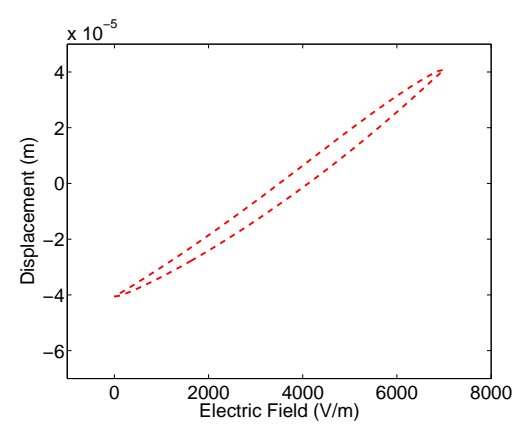

(a)

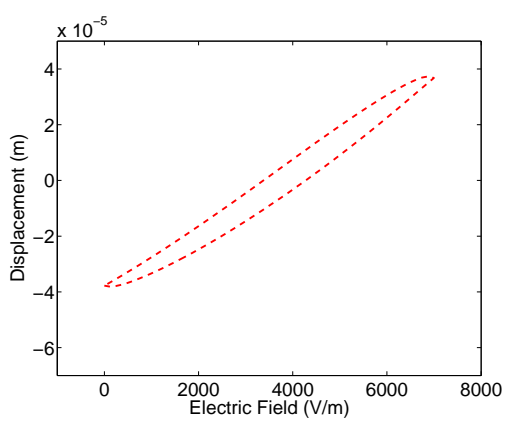

(b)

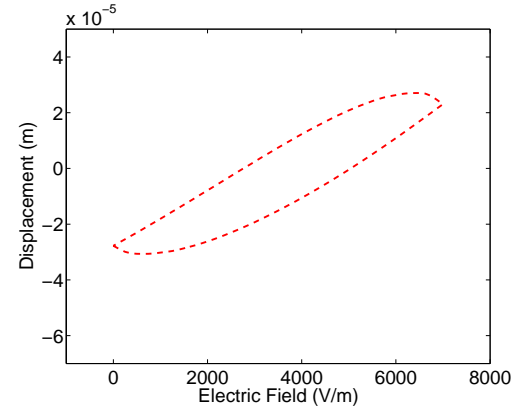

(c)

Figure 4: Frequency-dependent field-displacement behavior of a stacked PZT stage of the type depicted in Figure 2(a): sample rates of (a) $0.279 \mathrm{~Hz}$, (b) $5.58 \mathrm{~Hz}$, and (c) $27.9 \mathrm{~Hz}$.

characterization of hysteresis in certain stage constructs. Primary requirements for nonlinear hysteresis models for the PZT actuators in an AFM are (i) flexibility with regard to frequency-dependent hysteresis effects - the frameworks of $[5,30]$ are limited in this regard -, (ii) exact or approximate invertibility for linear control design, and (iii) sufficient efficiency for real-time implementation at the speeds required for present and future applications.

In this paper, we develop AFM transducer models, based on a homogenized energy framework for characterizing hysteresis and constitutive nonlinearities in ferroelectric materials, which meet these criteria. On Section 2, we summarize the framework developed in [33] for quantifying hysteresis in the field-polarization relation and develop constitutive equations which characterize the elastic and electromechanical behavior of the PZT material. These constitutive relations are employed in Section 3 to construct rod and shell models for the stages depicted in Figure 2, and the well-posedness of the models is established in Section 4. Numerical approximation techniques for the transducer models are summarized in Section 5, and the capability of the framework to quantify the biased and frequency-dependent hysteresis behavior of the transducers is illustrated in Section 6 through a comparison with the experimental data plotted in Figures 2 and 3.

With regard to criteria (ii) and (iii), the construction and experimental implementation of model inverses to linearize the nonlinear dynamics is demonstrated in [12]. Hence the models provide an effective framework for characterizing the hysteresis and nonlinear dynamics inherent to PZT-based nanopositioners in a manner which promotes stage and control design.

\section{Constitutive Relations}

In this section, we summarize the development of constitutive relations which quantify the nonlinear and hysteretic map between input fields $E$ and stresses $\sigma$ and the polarization $P$ and strains $\varepsilon$ generated in ferroelectric materials. These relations are developed in three steps. In the first, Helmholtz and Gibbs energy relations are constructed at the lattice level to quantify the local dependence of $P$ and $\varepsilon$ on $E$ and $\sigma$ for regimes in which relaxation due to thermal processes is either negligible or significant. In the second step of the development, material nonhomogeneities, polycrystallinity, and variable field effects are incorporated through the assumption that certain material properties are manifestations of underlying distributions rather than constants. Stochastic homogenization in this manner yields macroscopic models which quantify the bulk hysteretic $E$ - $P$ behavior measured in ferroelectric materials. Finally, necessary conditions associated with minimization of the Gibbs energy are invoked to obtain 1-D and 2-D constitutive relations quantifying the elastic and electromechanical behavior of the transducer materials. 


\subsection{Helmholtz and Gibbs Energy Relations}

As detailed in [33], an appropriate Helmholtz energy relation is

$$
\psi(P, \varepsilon)=\psi_{P}(P)+\frac{1}{2} Y \varepsilon^{2}-a_{1} \varepsilon P-a_{2} \varepsilon P^{2}
$$

where the component

$$
\psi_{P}(P)= \begin{cases}\frac{1}{2} \eta\left(P+P_{R}\right)^{2} & , P \leq-P_{I} \\ \frac{1}{2} \eta\left(P-P_{R}\right)^{2} & , P \geq P_{I} \\ \frac{1}{2} \eta\left(P_{I}-P_{R}\right)\left(\frac{P^{2}}{P_{I}}-P_{R}\right) & ,|P|<P_{I}\end{cases}
$$

quantifies the internal energy due to dipole processes. As shown in Figure $5, P_{I}$ is the positive inflection point which delineates the transition between stable and unstable regions, $P_{0}$ denotes the unstable equilibrium, and $P_{R}$ is the value of $P$ at which the positive local minimum of $\psi$ occurs. The parameter $\eta$ is the reciprocal of the slope of the $E-P$ relation after switching occurs. The second term on the right side of (1) quantifies the elastic energy whereas the third and fourth terms quantify electromechanical coupling effects. Here $Y$ denotes the Young's modulus and $a_{1}, a_{2}$ are electromechanical coupling coefficients.

The Gibbs energy relation

$$
G(E, \sigma, P, \varepsilon)=\psi_{P}(P)+\frac{1}{2} Y \varepsilon^{2}-a_{1} \varepsilon P-a_{2} \varepsilon P^{2}-E P-\sigma \varepsilon
$$

incorporates the elastic work $\sigma \varepsilon$ and electrostatic work $E P$. This provides the functional that is minimized or balanced with the relative thermal energy to provide local $E-P$ relations and global electromechanical constitutive equations. The reader is referred to $[25,27]$ for details regarding the manner through which the Gibbs energy incorporates the dependent variables $\varepsilon$ and $P$ in terms of the independent variables $\sigma$ and $E$.
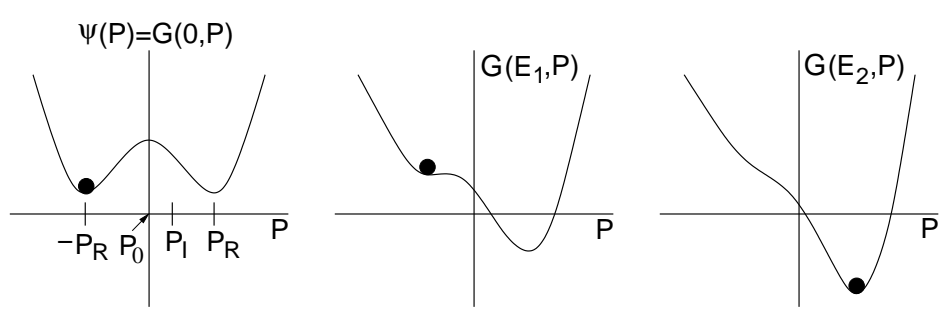

(a)
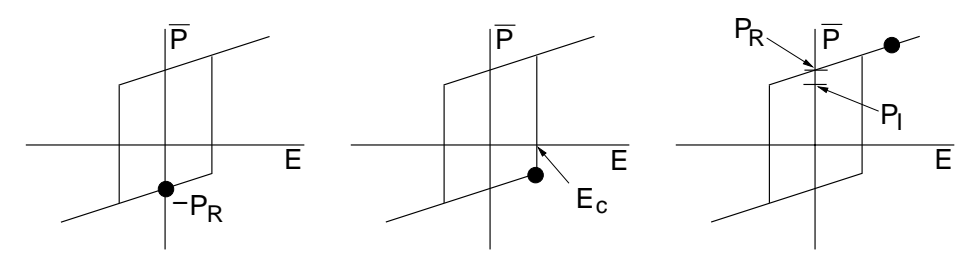

(b)

Figure 5: (a) Helmholtz energy $\psi$ and Gibbs energy $G$ for $\sigma=0$ and increasing fields $E$. (b) Switch in the local polarization $\bar{P}$ that occurs as $E$ is increased beyond the local coercive field $E_{c}$ given by (6) in the absence of thermal activation. 


\subsection{Polarization Kernel - Negligible Thermal Activation}

For operating regimes in which thermal activation is negligible, the local $E$ - $P$ relation is determined from the necessary conditions

$$
\frac{\partial G}{\partial P}=0, \frac{\partial^{2} G}{\partial P^{2}}>0 .
$$

For the piecewise quadratic functional (2), this yields a polarization kernel of the form

$$
\bar{P}(E)=\frac{E}{\eta-2 a_{2} \varepsilon}+\delta \frac{P_{R} \eta+\delta a_{1} \varepsilon}{\eta-2 a_{2} \varepsilon}
$$

where $\delta=1$ for positively oriented dipoles and $\delta=-1$ for those having negative orientation. To specify $\delta$, and hence $\bar{P}$, more specifically in terms of the initial dipole orientations and previous switches, we employ Preisach notation and take

$$
\left[\bar{P}\left(E ; E_{c}, \xi\right)\right](t)= \begin{cases}{\left[\bar{P}\left(E ; E_{c}, \xi\right)\right](0)} & , \tau(t)=\emptyset \\ \frac{E}{\eta}-P_{R} & , \tau(t) \neq \emptyset \text { and } E(\max \tau(t))=-E_{c} \\ \frac{E}{\eta}+P_{R} & , \tau(t) \neq \emptyset \text { and } E(\max \tau(t))=E_{c}\end{cases}
$$

Here

$$
\left[\bar{P}\left(E ; E_{c}, \xi\right)\right](0)= \begin{cases}\frac{E}{\eta}-P_{R} & , E(0) \leq-E_{c} \\ \xi & ,-E_{c}<E(0)<E_{c} \\ \frac{E}{\eta}+P_{R} & , E(0) \geq E_{c}\end{cases}
$$

defines initial kernel values in terms of the parameter $\xi=\frac{E_{0}}{\eta} \pm P_{R}, \emptyset$ designates the empty set, and the set of switching times is given by

$$
\tau(t)=\left\{t_{s} \in(0, t] \mid E\left(t_{s}\right)=-E_{c} \text { or } E\left(t_{s}\right)=E_{c}\right\} .
$$

The local coercive field

$$
E_{c}=\eta\left(P_{R}-P_{I}\right)
$$

quantifies the field at which the negative well ceases to exist and hence a dipole switch occurs. To illustrate, the condition $\tau \neq \emptyset$ and $E(\max \tau(t))=E_{c}$ designates that switching has occurred and the last switch was at $E_{c}$; hence the local polarization is $\left[\bar{P}\left(E ; E_{c}, \xi\right)\right](t)=\frac{E(t)}{\eta}+P_{R}$.

Remark 1 For the drive levels employed for nanopositioning, the stress effects on the polarization are typically negligible which motivates taking $\varepsilon=0$ in (3)-(5). Hence the relations

$$
\begin{aligned}
& \bar{P}(E)=\frac{1}{\eta} E+P_{R} \delta \\
& {\left[\bar{P}\left(E ; E_{c}, \xi\right)\right](t)= \begin{cases}{\left[\bar{P}\left(E ; E_{c}, \xi\right)\right](0)} & , \tau(t)=\emptyset \\
\frac{E}{\eta}-P_{R} & , \tau(t) \neq \emptyset \text { and } E(\max \tau(t))=-E_{c} \\
\frac{E}{\eta}+P_{R} & , \tau(t) \neq \emptyset \text { and } E(\max \tau(t))=E_{c}\end{cases} }
\end{aligned}
$$

and

$$
\left[\bar{P}\left(E ; E_{c}, \xi\right)\right](0)= \begin{cases}\frac{E}{\eta}-P_{R} & , E(0) \leq-E_{c} \\ \xi & ,-E_{c}<E(0)<E_{c} \\ \frac{E}{\eta}+P_{R} & , E(0) \geq E_{c}\end{cases}
$$

are usually employed when characterizing AFM stages. 


\subsection{Polarization Kernel — Thermal Activation}

If thermal relaxation is significant, the Gibbs energy $G$ and relative thermal energy $k T / V$ are balanced through the Boltzmann relation

$$
\mu(G)=C e^{-G V / k T} .
$$

Here $k$ is Boltzmann's constant, $V$ denotes a reference volume chosen to ensure physical relaxation behavior, and $C$ is chosen to ensure integration to unity for the complete set of admissible inputs. As detailed in [25, 33], this yields the local polarization relation

$$
\bar{P}=x_{+}\left\langle P_{+}\right\rangle+x_{-}\left\langle P_{-}\right\rangle .
$$

The fractions $x_{+}$and $x_{-}$of positively and negatively oriented dipoles are quantified by the differential equations

$$
\begin{aligned}
& \dot{x}_{+}=-p_{+-} x_{+}+p_{-+} x_{-} \\
& \dot{x}_{-}=-p_{-+} x_{-}+p_{+-} x_{+}
\end{aligned}
$$

which can be simplified to

$$
\dot{x}_{+}=-p_{+-} x_{+}+p_{-+}\left(1-x_{+}\right)
$$

through the identity

$$
\dot{x}_{+}+\dot{x}_{-}=1 .
$$

The expected polarizations due to positively and negatively oriented dipoles are

$$
\left\langle P_{+}\right\rangle=\frac{\int_{P_{I}}^{\infty} P e^{-G(E, P) V / k T} d P}{\int_{P_{I}}^{\infty} e^{-G(E, P) V / k T} d P} \quad, \quad\left\langle P_{-}\right\rangle=\frac{\int_{-\infty}^{-P_{I}} P e^{-G(E, P) V / k T} d P}{\int_{-\infty}^{-P_{I}} e^{-G(E, P) V / k T} d P}
$$

where the denominator results from the evaluation of $C$ in (8). The likelihoods of switching from positive to negative, and conversely, are given by

$$
p_{+-}=\frac{1}{\mathcal{T}(T)} \frac{\int_{P_{I}-\epsilon}^{P_{I}} e^{-G(E, P) V / k T} d P}{\int_{P_{I}-\epsilon}^{\infty} e^{-G(E, P) V / k T} d P} \quad, \quad p_{-+}=\frac{1}{\mathcal{T}(T)} \frac{\int_{-P_{I}}^{-P_{I}+\epsilon} e^{-G(E, P) V / k T} d P}{\int_{-\infty}^{-P_{I}+\epsilon} e^{-G(E, P) V / k T} d P}
$$

where $\epsilon$ is taken to be a small positive constant. The relaxation time $\mathcal{T}$ is the reciprocal of the frequency at which dipoles attempt to switch. It is proven in $[25,33]$ that $\bar{P}$ given by (9) converges to the local polarization ( 7$)$ in the limit $k T / V \rightarrow 0$ of negligible thermal activation.

Remark 2 In (10) and (11), we use the notation $G(E, P)$ to indicate that we take $\varepsilon=\sigma=0$ in (2) in accordance with the assumption that stress effects on the polarization are negligible at the drive levels employed in AFM stages. 


\subsection{Macroscopic Polarization Model}

The local polarization relations (7) and (9) exhibit the behavior depicted in Figure 6 and provide reasonable characterization of the $E-P$ behavior of certain single crystal compounds. However, to incorporate the effects of material and stress nonhomogeneities, polycrystallinity, and variable effective fields $E_{e}=E+E_{I}$, we assume that the interaction field $E_{I}$ and local coercive field $E_{c}$ given by (6) are manifestations of underlying distributions rather than constants. If we designate the associated densities by $\nu_{1}$ and $\nu_{2}$, the macroscopic field-polarization behavior is quantified by the relation

$$
[P(E)](t)=\int_{0}^{\infty} \int_{-\infty}^{\infty} \nu_{1}\left(E_{c}\right) \nu_{2}\left(E_{I}\right)\left[\bar{P}\left(E+E_{I} ; E_{c}, \xi\right)\right](t) d E_{I} d E_{c}
$$

where the kernel $\bar{P}$ is given by (7) or (9).

As detailed in $[25,27]$, the densities $\nu_{1}$ and $\nu_{2}$ are assumed to satisfy the physical criteria

$$
\begin{array}{ll}
\text { (i) } & \nu_{1}(x) \text { defined for } x>0, \\
\text { (ii) } & \nu_{2}(-x)=\nu_{2}(x), \\
\text { (iii) } & \left|\nu_{1}(x)\right| \leq c_{1} e^{-a_{1} x} \\
& \left|\nu_{2}(x)\right| \leq c_{2} e^{-a_{2}|x|}
\end{array}
$$

for positive $c_{1}, a_{1}, c_{2}, a_{2}$. The restricted domain in (i) reflects the fact that the coercive field $E_{c}$ is positive whereas the symmetry enforced in the interaction field through (ii) yields the symmetry observed in low-field Rayleigh loops. Hypothesis (iii) incorporates the physical observation that the coercive and interaction fields decay as a function of distance and guarantees that integration against the piecewise linear kernel yields finite polarization values.

Approximation of (12) through Gaussian quadrature techniques yields the approximate relation

$$
[P(E)](t)=\sum_{i=1}^{N_{i}} \sum_{j=1}^{N_{j}} \nu_{1}\left(E_{c_{i}}\right) \nu_{2}\left(E_{I_{j}}\right)\left[\bar{P}\left(E_{I_{j}}+E ; E_{c_{i}}, \xi_{j}\right)\right](t) v_{i} w_{j}
$$

where $E_{I_{j}}, E_{c_{i}}$ denote the abscissas associated with respective quadrature formulae and $v_{i}, w_{j}$ are the respective weights - e.g., see [25]. Algorithms used to implement the approximate polarization model (14) are provided in $[25,33]$.

Techniques for identifying the densities $\nu_{1}$ and $\nu_{2}$ are illustrated in [25, 27]. For certain applications, reasonable accuracy is provided by a priori functions satisfying the physical criteria (13) and having a small number of parameters to be estimated through least squares fits to data - e.g., variances and means in normal and lognormal relations. For more general applications requiring high accuracy for a wide range of operating conditions, the $N_{i}+N_{j}$ discretized density values $\nu_{1}\left(E_{c_{i}}\right)$ and $\nu_{2}\left(E_{I_{j}}\right)$ can be estimated through least squares techniques.

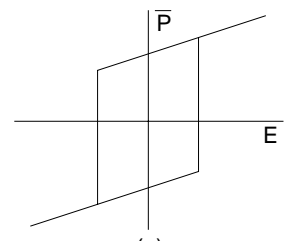

(a)

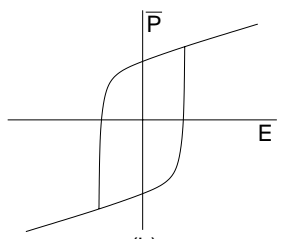

(b)

Figure 6: Hysteron provided by (a) the relation (7) with negligible thermal relaxation, and (b) the relation (9) which incorporates relaxation mechanisms. 
Remark 3 From the perspective of both numerical and experimental implementation and the establishment of the well-posedness of resulting transducer models, it is important to quantify the regularity between input fields and the polization predicted by (12). In Appendix A, it is established that $P$ given by (12) is continuous with respect to $E$.

\subsection{Constitutive Relations}

To obtain a elastic constitutive relations, the equilibrium condition

$$
\frac{\partial G}{\partial \varepsilon}=0
$$

is invoked to obtain

$$
\sigma=Y \varepsilon-a_{1} P-a_{2} P^{2}
$$

which reduces to Hooke's law when $P=0$. To incorporate internal damping, we posit that in the absence of electromechanical effects, stress is proportional to a linear combination of strain and strain rate (Kelvin-Voigt damping hypothesis). Finally, we note that the PZT stage mechanisms are poled and hence operate about the remanence polarization $P=P_{R}$ rather than the depoled state $P=0$. When combined with the polarization model (12), this yields the 1-D constitutive relations

$$
\begin{aligned}
& \sigma=Y \varepsilon+C \dot{\varepsilon}-a_{1}\left(P-P_{R}\right)-a_{2}\left(P-P_{R}\right)^{2} \\
& {[P(E)](t)=\int_{0}^{\infty} \int_{-\infty}^{\infty} \nu_{1}\left(E_{c}\right) \nu_{2}\left(E_{I}\right)\left[\bar{P}\left(E+E_{I} ; E_{c}, \xi\right)\right](t) d E_{I} d E_{c}}
\end{aligned}
$$

where $C$ is the Kelvin-Voigt damping coefficient. These relations are employed when constructing rod models to characterize the hysteretic dynamics shown in Figures 3 and 4 for the stacked actuators employed in the stage construction depicted in Figure 2(a).

The constitutive behavior of the PZT shell depicted in Figure 2(b) differs from that of the rod in two fundamental aspects: (i) the longitudinal actuation is due to $d_{31}$ rather than $d_{33}$ mechanisms, and (ii) longitudinal and circumferential stresses and strains are coupled due to the curvature. To designate the coupled material behavior, we let $\varepsilon_{x}, \sigma_{x}$ and $\varepsilon_{\theta}, \sigma_{\theta}$ respectively denote the normal strains and stresses in the longitudinal and circumferential directions and we denote shear strains and stresses by $e_{x \theta}$ and $\sigma_{x \theta}$. Finally, we let $\nu$ denote the Poisson ratio for the material. The resulting 2-D constitutive relations

$$
\begin{aligned}
& \sigma_{x}=\frac{Y}{1-\nu^{2}}\left(\varepsilon_{x}+\nu \varepsilon_{\theta}\right)+\frac{C}{1-\nu^{2}}\left(\dot{\varepsilon}_{x}+\nu \dot{\varepsilon}_{\theta}\right)-\frac{1}{1-\nu}\left[a_{1}\left(P-P_{R}\right)+a_{2}\left(P-P_{R}\right)^{2}\right] \\
& \sigma_{\theta}=\frac{Y}{1-\nu^{2}}\left(\varepsilon_{\theta}+\nu \varepsilon_{x}\right)+\frac{C}{1-\nu^{2}}\left(\dot{\varepsilon}_{\theta}+\nu \dot{\varepsilon}_{x}\right)-\frac{1}{1-\nu}\left[a_{1}\left(P-P_{R}\right)+a_{2}\left(P-P_{R}\right)^{2}\right] \\
& \sigma_{x \theta}=\frac{Y}{2(1+\nu)} \varepsilon_{x \theta}+\frac{C}{2(1+\nu)} \dot{\varepsilon}_{x \theta} \\
& {[P(E)](t)=\int_{0}^{\infty} \int_{-\infty}^{\infty} \nu_{1}\left(E_{c}\right) \nu_{2}\left(E_{I}\right)\left[\bar{P}\left(E+E_{I} ; E_{c}, \xi\right)\right](t) d E_{I} d E_{c}}
\end{aligned}
$$

are employed when constructing transducer models for cylindrical nanopositioning stages. 


\section{Transducer Models for Stacked and Cylindrical AFM Stages}

We now employ the 1-D constitutive relation (15) and 2-D relation (16) to construct models for the stacked and cylindrical AFM stages depicted in Figure 2. For the stacked actuator, we consider two frameworks: (i) a distributed PDE model which quantifies displacements along the rod length as a function of the input field, and (ii) a lumped model which exploits the assumption of uniform stresses and fields along the rod length to motivate an ODE quantifying displacements only at the rod end. A comparison between characterization capabilities provided by the two frameworks is provided in Section 6. For the cylindrical shell design, we summarize a Donnell-Mushtari model which quantifies vertical motion provided by the $z$-component of the stage depicted in Figure 2(b).

\subsection{Rod Model for the Stacked Actuator}

\section{Distributed Rod Model}

We consider first the development of a distributed rod model which quantifies the displacement $u(t, x)$ along the rod length. In accordance with present stage design, one end of the rod is assumed fixed while the other encounters resistance due to the connecting mechanisms. We assume that this latter contribution can be modeled as a damped elastic system with mass $m_{\ell}$, stiffness $k_{\ell}$ and damping coefficient $c_{\ell}$. The density, cross-sectional area and length of the rod are denoted by $\rho, A$ and $\ell$ and, in accordance with (15), the Young's modulus and Kelvin-Voigt damping parameter are denoted by $Y$ and $C$.

Force balancing yields the relation

$$
\rho A \frac{\partial^{2} u}{\partial t^{2}}=\frac{\partial \mathcal{N}}{\partial x}
$$

where the resultant $N=\int_{A} \sigma d A$ is given by

$$
N=Y A \frac{\partial u}{\partial x}+C A \frac{\partial^{2} u}{\partial x \partial t}-a_{1}\left[P(E)-P_{R}\right]-a_{2}\left[P(E)-P_{R}\right]^{2}
$$

once the linear relation $\varepsilon=\frac{\partial u}{\partial x}$ is employed for the strains in (15). The nonlinear and hysteretic map between input fields $E$ and the polarization $P$ is specified by (12). The fixed-end condition yields $u(t, 0)=0$ and balancing forces at $x=\ell$ yields the energy dissipating end condition

$$
N(t, \ell)=-k_{L} u(t, \ell)-c_{L} \frac{\partial u}{\partial t}(t, \ell)-M_{L} \frac{\partial^{2} u}{\partial t^{2}}(t, \ell) .
$$

Finally, initial conditions are taken to be $u(0, x)=u_{0}(x)$ and $\frac{\partial u}{\partial t}(0, x)=u_{1}(x)$. This provides a strong formulation of the stacked actuator model.

To define a weak or variational form of the model which is appropriate for well-posedness analysis, approximation, or control design, states $z=(u(\cdot), u(\ell))$ are considered in the state space $X=$

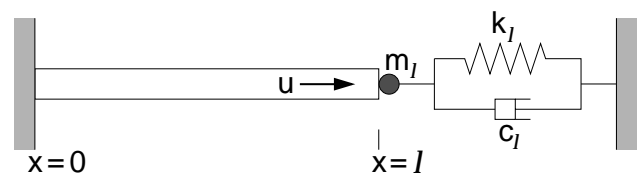

Figure 7: Rod of length $\ell$ and cross-sectional area $A$ with a fixed end at $x=0$ and energy dissipating boundary conditions at $x=\ell$. 
$L^{2}(0, \ell) \times \mathbb{R}$ with the inner product

$$
\left\langle\Phi_{1}, \Phi_{2}\right\rangle_{X}=\int_{0}^{\ell} \rho A \phi_{1} \phi_{2} d x+m_{\ell} \varphi_{1} \varphi_{2}
$$

where $\Phi_{1}=\left(\phi_{1}, \varphi_{1}\right), \Phi_{2}=\left(\phi_{2}, \varphi_{2}\right)$ with $\varphi_{1}=\phi_{1}(\ell), \varphi_{2}=\phi_{1}(\ell)$. The space of test functions is taken to be

$$
V=\left\{\Phi=(\phi, \varphi) \in X \mid \phi \in H^{1}(0, \ell), \phi(0)=0, \phi(\ell)=\varphi\right\}
$$

with the inner product

$$
\left\langle\Phi_{1}, \Phi_{2}\right\rangle_{V}=\int_{0}^{\ell} Y A \phi_{1}^{\prime} \phi_{2}^{\prime} d x+k_{\ell} \varphi_{1} \varphi_{2} .
$$

Multiplication by $\phi \in H_{0}^{1}(0, \ell)=\left\{\phi \in H^{1}(0, \ell) \mid \phi(0)=0\right\}$ and integration by parts in space yields the weak model formulation

$$
\begin{aligned}
\int_{0}^{\ell} \rho A & \frac{\partial^{2} u}{\partial t^{2}} \phi d x+\int_{0}^{\ell}\left[Y A \frac{\partial u}{\partial x}+C A \frac{\partial^{2} u}{\partial x \partial t}\right] \frac{d \phi}{d x} d x=\int_{0}^{\ell} f \phi d x \\
& +A\left[a_{1}\left(P-P_{R}\right)+a_{2}\left(P-P_{R}\right)^{2}\right] \int_{0}^{\ell} \frac{d \phi}{d x} d x-\left[k_{\ell} u(t, \ell)+c_{\ell} \frac{\partial u}{\partial t}(t, \ell)+m_{\ell} \frac{\partial^{2} u}{\partial t^{2}}(t, \ell)\right] \phi(\ell)
\end{aligned}
$$

which must be satisfied for all $\phi \in V$.

\section{Lumped Rod Model}

The assumption that fields and stresses are uniform along the rod length motivates the conclusion that strains (relative displacements) also exhibit negligible $x$-dependence. Since the position of the sample is dictated by the position of the rod tip at $x=\ell$, this motivates the development of a lumped model which quantifies $u_{\ell}(t)=u(t, \ell)$.

From the assumption of uniform strains along the rod length, we take

$$
\varepsilon(t)=\frac{u_{\ell}(t)}{\ell}
$$

in (15). Balancing the forces $\sigma A$ for the rod with those of the restoring mechanism yields the lumped model

$$
\begin{aligned}
\rho A \ell \frac{d^{2} u_{\ell}}{d t^{2}}(t)+\frac{C A}{\ell} \frac{d u_{\ell}}{d t}(t)+\frac{Y A}{\ell} u_{\ell}(t)= & -m_{\ell} \frac{d^{2} u_{\ell}}{d t^{2}}(t)-c_{\ell} \frac{d u_{\ell}}{d t}(t)-k u_{\ell}(t) \\
& +A a_{1}\left[P(E(t))-P_{R}\right]+A a_{2}\left[P(E(t))-P_{R}\right]^{2}
\end{aligned}
$$

or, equivalently,

$$
m \frac{d^{2} u_{\ell}}{d t^{2}}(t)+c \frac{d u_{\ell}}{d t}(t)+k u_{\ell}(t)=\widetilde{a}_{1}\left[P(E(t))-P_{R}\right]+\widetilde{a}_{2}\left[P(E(t))-P_{R}\right]^{2}
$$

where

$$
m=\rho A \ell+m_{\ell} \quad, \quad c=\frac{C A}{\ell}+c_{\ell} \quad, \quad k=\frac{Y A}{\ell}+k_{\ell} \quad, \quad \widetilde{a}_{1}=A a_{1} \quad, \quad \tilde{a}_{2}=A a_{2}
$$

and the initial conditions are $u_{\ell}(0)=u_{0}$ and $\frac{d u_{\ell}}{d t}(0)=u_{1}$. The polarization $P$ is specified by the model (12) or discretized model (14). 
The model can also be written as the first-order system

$$
\begin{aligned}
\dot{\vec{u}}_{\ell}(t) & =A \vec{u}_{\ell}(t)+\overrightarrow{\mathcal{P}}(E(t)) \\
\vec{u}_{\ell}(0) & =\vec{u}_{0}
\end{aligned}
$$

where $\vec{u}_{\ell}(t)=\left[u_{\ell}(t), \dot{u}_{\ell}(t)\right]^{T}, \vec{u}_{\ell}(0)=\left[u_{0}, u_{1}\right]^{T}$ and

$$
A=\left[\begin{array}{cc}
0 & 1 \\
-k / m & -c / m
\end{array}\right] \quad, \quad \overrightarrow{\mathcal{P}}(E(t))=\frac{1}{m}\left[\widetilde{a}_{1}\left(P(E(t))-P_{R}\right)+\widetilde{a}_{2}\left(P(E(t))-P_{R}\right)^{2}\right]\left[\begin{array}{l}
0 \\
1
\end{array}\right] .
$$

\subsection{Cylindrical Shell Model}

To quantify the dynamics of the cylindrical stage depicted in Figure 2(b), we construct a linear shell model with nonlinear inputs quantified by the 2-D constitutive relation (16). We focus on the actuator employed for transverse displacements since real-time control of this component is required to maintain constant forces between the sample and micro-cantilever. The mass of the shell employed for horizontal translation is combined with the mass of the sample to provide an inertial force acting on the free end of the vertical actuator.

For modeling purposes, we assume that the shell has length $\ell$, thickness $h$, and radius $R$. The axial direction is specified along the $x$-axis and the longitudinal, circumferential and transverse displacements are respectively denoted by $u, v$ and $w$ as depicted in Figure 8 . The density is designated by $\rho$ and the region occupied by the reference or middle surface of the shell is specified by $\Gamma_{0}=[0, \ell] \times[0,2 \pi]$. In accordance with the constitutive relations (16), $Y, C$ and $\nu$ denote the Young's modulus, Kelvin-Voigt damping coefficient and Poisson ratio for the material. We point out that $\varepsilon_{x}, \varepsilon_{\theta}$ and $\varepsilon_{x \theta}$ in (16) denote strains at points throughout the shell thickness whereas 2-D shell models are formulated in terms of strains $e_{x}, e_{\theta}$ and $e_{x \theta}$ in the reference surface of the shell. The relationship between the two is established through the assumption that displacements are linear through the shell thickness which comprises one of the fundamental tenets of linear shell theory $[2,25]$.

We consider the case in which the bottom edge of the shell $(x=0)$ is clamped and the opposite end $(x=\ell)$ is acted upon only by the inertial force associated with the combined mass $m$ of the $x-y$ actuator and the sample.

As detailed in $[2,25]$, force and moment balancing yield the Donnell-Mushtari shell equations

$$
\begin{aligned}
& R \rho h \frac{\partial^{2} u}{\partial t^{2}}-R \frac{\partial N_{x}}{\partial x}-\frac{\partial N_{x \theta}}{\partial \theta}=0 \\
& R \rho h \frac{\partial^{2} v}{\partial t^{2}}-\frac{\partial N_{\theta}}{\partial \theta}-R \frac{\partial N_{x \theta}}{\partial x}=0 \\
& R \rho h \frac{\partial^{2} w}{\partial t^{2}}-R \frac{\partial^{2} M_{x}}{\partial x^{2}}-\frac{1}{R} \frac{\partial^{2} M_{\theta}}{\partial \theta^{2}}-2 \frac{M_{x \theta}}{\partial x \partial \theta}+N_{\theta}=0
\end{aligned}
$$

where the force and moment resultants are

$$
\begin{aligned}
& N_{x}=\frac{Y h}{1-\nu^{2}}\left(e_{x}+\nu e_{\theta}\right)+\frac{C h}{1-\nu^{2}}\left(\dot{e}_{x}+\nu \dot{e}_{\theta}\right)-\frac{h}{1-\nu}\left[a_{1}\left(P-P_{R}\right)+a_{2}\left(P-P_{R}\right)^{2}\right] \\
& N_{\theta}=\frac{Y h}{1-\nu^{2}}\left(e_{\theta}+\nu e_{x}\right)+\frac{C h}{1-\nu^{2}}\left(\dot{e}_{\theta}+\nu \dot{e}_{x}\right)-\frac{h}{1-\nu}\left[a_{1}\left(P-P_{R}\right)+a_{2}\left(P-P_{R}\right)^{2}\right] \\
& N_{x \theta}=\frac{Y h}{2(1+\nu)} e_{x \theta}+\frac{C h}{2(1+\nu)} \dot{e}_{x \theta}
\end{aligned}
$$




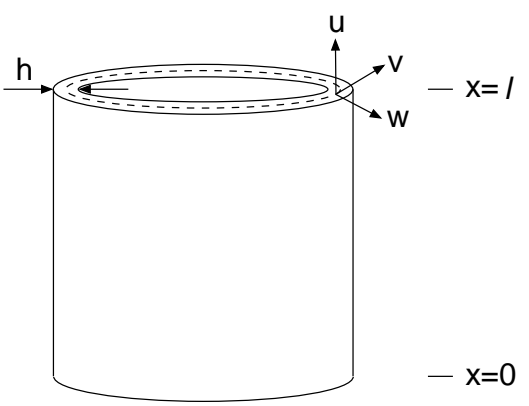

Figure 8: Orientation of the shell geometry used when quantifying the longitudinal, circumferential and transverse displacements $u, v$ and $w$.

and

$$
\begin{aligned}
& M_{x}=\frac{Y h^{3}}{12\left(1-\nu^{2}\right)}\left(\kappa_{x}+\nu \kappa_{\theta}\right)+\frac{C h^{3}}{12\left(1-\nu^{2}\right)}\left(\dot{\kappa}_{x}+\nu \dot{\kappa}_{\theta}\right) \\
& M_{\theta}=\frac{Y h^{3}}{12\left(1-\nu^{2}\right)}\left(\kappa_{\theta}+\nu \kappa_{x}\right)+\frac{C h^{3}}{12\left(1-\nu^{2}\right)}\left(\dot{\kappa}_{\theta}+\nu \dot{\kappa}_{x}\right) \\
& M_{x \theta}=\frac{Y h^{3}}{24(1+\nu)} \kappa_{x \theta}+\frac{C h^{3}}{24(1+\nu)} \dot{\kappa}_{x \theta} .
\end{aligned}
$$

The midsurface strains and changes in curvature are

$$
\begin{aligned}
& e_{x}=\frac{\partial u}{\partial x} \quad, \quad e_{\theta}=\frac{1}{R} \frac{\partial v}{\partial \theta}+\frac{w}{R} \quad, \quad e_{x \theta}=\frac{\partial v}{\partial x}+\frac{1}{R} \frac{\partial u}{\partial \theta} \\
& \kappa_{x}=-\frac{\partial^{2} w}{\partial x^{2}} \quad, \quad \kappa_{\theta}=-\frac{1}{R^{2}} \frac{\partial^{2} w}{\partial \theta^{2}} \quad, \quad \kappa_{x \theta}=-\frac{2}{R} \frac{\partial^{2} w}{\partial x \partial \theta} .
\end{aligned}
$$

The boundary conditions for the fixed-end at $x=0$ are taken to be

$$
u=v=w=\frac{\partial w}{\partial x}=0
$$

whereas the conditions

$$
\begin{array}{ll}
N_{x}=-m \frac{\partial^{2} u}{\partial t^{2}} & , \quad N_{x \theta}+\frac{M_{x \theta}}{R}=0 \\
Q_{x}+\frac{1}{R} \frac{\partial M_{x \theta}}{\partial \theta}=0 & , \quad M_{x}=0
\end{array}
$$

are employed at $x=\ell$. The first resultant condition incorporates the inertial force due to the mass $m$ of the PZT actuator employed for $x-y$ translation along with the mass of the sample.

To reduce smoothness requirements for approximation and eliminate the Dirac behavior of external inputs at $x=\ell$, we also consider a weak formulation of the model. The state is taken to be $z=(u(\cdot, \cdot), v(\cdot, \cdot), w(\cdot, \cdot), u(\ell, \cdot))$ in the state space

$$
X=L^{2}(\Omega) \times L^{2}(\Omega) \times L^{2}(\Omega) \times L^{2}(0,2 \pi)
$$

where

$$
\Omega=[0, \ell] \times[0,2 \pi]
$$


denotes the shell region. The space of test functions is specified as

$$
V=\left\{\Phi=\left(\phi_{1}, \phi_{2}, \phi_{3}, \eta\right) \in X \mid \phi_{1} \in H_{0}^{1}(\Omega), \phi_{2} \in H_{0}^{1}(\Omega), \phi_{3} \in H_{0}^{2}(\Omega)\right\}
$$

where $\eta(\theta)=\phi_{1}(\ell, \theta)$ and

$$
\begin{aligned}
& H_{0}^{1}(\Omega)=\left\{\phi \in H^{1}(\Omega) \mid \phi(0, \theta)=0\right\} \\
& H_{0}^{2}(\Omega)=\left\{\phi \in H^{2}(\Omega) \mid \phi(0, \theta)=\phi^{\prime}(0, \theta)=0\right\} .
\end{aligned}
$$

Through either variation principles — e.g., see [2] — or integration by parts, one obtains the weak formulation of the thin shell model,

$$
\begin{aligned}
& \int_{\Omega}\left\{R \rho h \frac{\partial^{2} u}{\partial t^{2}} \phi_{1}+R N_{x} \frac{\partial \phi_{1}}{\partial x}+N_{x \theta} \frac{\partial \phi_{1}}{\partial \theta}\right\} d \omega=0 \\
& \int_{\Omega}\left\{R \rho h \frac{\partial^{2} v}{\partial t^{2}} \phi_{2}+N_{\theta} \frac{\partial \phi_{2}}{\partial \theta}+R N_{x \theta} \frac{\partial \phi_{2}}{\partial x}\right\} d \omega=0 \\
& \int_{\Omega}\left\{R \rho h \frac{\partial^{2} w}{\partial t^{2}} \phi_{3}-R M_{x} \frac{\partial^{2} \phi_{3}}{\partial x^{2}}-2 M_{x \theta} \frac{\partial^{2} \phi_{3}}{\partial x \partial \theta}-\frac{1}{R} M_{\theta} \frac{\partial^{2} \phi_{3}}{\partial \theta^{2}}+N_{\theta} \phi_{3}\right\} d \omega=0
\end{aligned}
$$

which must be satisfied for all $\Phi \in V$. The resultants are given by (25) and (26) with midsurface strains and changes in curvature designated in (27).

Remark 4 It is noted that the $d_{31}$ poling, used to generate vertical motion in the stage, produces no polarization contributions to the moments. However, transverse displacements $w$ in the shell model are generated by the $N_{\theta}$ resultant in the $w$ relation and hence all three components of the displacement are coupled.

\section{Model Well-Posedness}

\subsection{Rod Model}

To provide a framework which facilitates the establishment of criteria which guarantee the existence of a unique solution to the distributed rod model with nonlinear inputs, we consider a Hilbert space formulation of the weak model formulation (20) with the state and test function spaces

$$
\begin{aligned}
& X=L^{2}(0, \ell) \times \mathbb{R} \\
& V=\left\{\Phi=(\phi, \varphi) \in X \mid \phi \in H^{1}(0, \ell), \phi(0)=0, \phi(\ell)=\varphi\right\}
\end{aligned}
$$

and inner products

$$
\begin{aligned}
\left\langle\Phi_{1}, \Phi_{2}\right\rangle_{X} & =\int_{0}^{\ell} \rho A \phi_{1} \phi_{2} d x+m_{\ell} \varphi_{1} \varphi_{2} \\
\left\langle\Phi_{1}, \Phi_{2}\right\rangle_{V} & =\int_{0}^{\ell} Y A \phi_{1}^{\prime} \phi_{2}^{\prime} d x+k_{\ell} \varphi_{1} \varphi_{2}
\end{aligned}
$$

where $\Phi_{1}=\left(\phi_{1}, \varphi_{1}\right), \Phi_{2}=\left(\phi_{2}, \varphi_{2}\right)$ with $\varphi_{1}=\phi_{1}(\ell), \varphi_{2}=\phi_{1}(\ell)$.

It is observed that $V$ is densely and continuously embedded in $X$ with $|\Phi|_{X} \leq c|\Phi|_{V}$; this is expressed by $V \hookrightarrow X$. Moreover, when one defines conjugate dual spaces $X^{*}$ and $V^{*}-$ e.g., $V^{*}$ denotes the linear space of all conjugate linear continuous functionals on $V$ - two observations are 
important: (i) $X^{*}$ can be identified with $X$ through the Riesz map, and (ii) $X^{*} \hookrightarrow V^{*}$. Hence the two spaces comprise what is termed a Gelfand triple $V \hookrightarrow X \cong X^{*} \hookrightarrow V^{*}$ with pivot space $X$ and duality pairing (duality product) $\langle\cdot, \cdot\rangle_{V^{*}, V}$. The latter is defined as the extension by continuity of the inner product $\langle\cdot, \cdot\rangle_{X}$ from $V \times X$ to $V^{*} \times X$. Hence elements $v^{*} \in V^{*}$ have the representation $v^{*}(v)=\left\langle v^{*}, v\right\rangle_{V^{*}, V}$.

We now define the stiffness and damping sesquilinear forms $\sigma_{i}: V \times V \rightarrow \mathbb{C}, i=1,2$, by

$$
\begin{aligned}
& \sigma_{1}\left(\Phi_{1}, \Phi_{2}\right)=\left\langle\Phi_{1}, \Phi_{2}\right\rangle_{V} \\
& \sigma_{2}\left(\Phi_{1}, \Phi_{2}\right)=\int_{0}^{\ell} C A \phi_{1}^{\prime} \phi_{2}^{\prime} d x+c_{\ell} \varphi_{1} \varphi_{2} .
\end{aligned}
$$

It can be directly verified that the stiffness form satisfies

$$
\begin{array}{ll}
\left|\sigma_{1}\left(\Phi_{1}, \Phi_{2}\right)\right| \leq c_{1}\left|\Phi_{1}\right|_{V}\left|\Phi_{2}\right|_{V}, \text { for some } c_{1} \in \mathbb{R} & \text { (Bounded) } \\
\operatorname{Re} \sigma_{1}\left(\Phi_{1}, \Phi_{1}\right) \geq c_{2}\left|\Phi_{1}\right|_{V}^{2}, \text { for some } c_{2}>0 & \text { (V-Elliptic) } \\
\sigma_{1}\left(\Phi_{1}, \Phi_{2}\right)=\overline{\sigma_{1}\left(\Phi_{2}, \Phi_{1}\right)} & \text { (Symmetric) }
\end{array}
$$

for all $\psi, \phi \in V$. Moreover, the damping term $\sigma_{2}$ satisfies

$$
\begin{array}{ll}
\left|\sigma_{2}\left(\Phi_{1}, \Phi_{2}\right)\right| \leq c_{3}\left|\Phi_{1}\right|_{V}\left|\Phi_{2}\right|_{V}, \text { for some } c_{3} \in \mathbb{R} & \text { (Bounded) } \\
\operatorname{Re} \sigma_{2}\left(\Phi_{1}, \Phi_{1}\right) \geq c_{4}\left|\Phi_{1}\right|_{V}^{2}, \text { for some } c_{4}>0 & (V \text {-Elliptic). }
\end{array}
$$

The input space is taken to be the Hilbert space $U=\mathbb{R}$ and the input operator $B: U \rightarrow V^{*}$ is defined by

$$
\langle[B(E)](t), \Phi\rangle_{V^{*}, V}=\left[a_{1}\left(P(E(t))-P_{R}\right)+a_{2}\left(P(E(t))-P_{R}\right)^{2}\right] \int_{0}^{\ell} \phi^{\prime} d x
$$

for $\Phi=(\phi, \varphi)$ with $\varphi=\phi(\ell)$. It is observed that $B$ can be expressed as

$$
[B(E)](t)=[b(E)](t) \cdot g \quad, g \in V^{*}
$$

where

$$
\begin{aligned}
& {[b(E)](t)=\left(P(E(t))-P_{R}\right)+a_{2}\left(P(E(t))-P_{R}\right)^{2}} \\
& g(\Phi)=\int_{0}^{\ell} \phi^{\prime} d x .
\end{aligned}
$$

The model (20) can then be written in the abstract variational formulation

$$
\begin{aligned}
& \langle\ddot{u}(t), \Phi\rangle_{V^{*}, V}+\sigma_{2}(\dot{u}(t), \Phi)+\sigma_{1}(u(t), \Phi)=\langle[B(E)](t), \Phi\rangle_{V^{*}, V} \\
& u(0)=u_{0} \quad, \quad \dot{u}(0)=u_{1}
\end{aligned}
$$

for all $\Phi \in V$.

Alternatively, one can define the operators $A_{i} \in \mathcal{L}\left(V, V^{*}\right), i=1,2$, by

$$
\left\langle A_{i} \Phi_{1}, \Phi_{2}\right\rangle_{V^{*}, V}=\sigma_{i}\left(\Phi_{1}, \Phi_{2}\right)
$$

and formulate the model in operator form as

$$
\begin{aligned}
& \ddot{u}(t)+A_{2} \dot{u}(t)+A_{1} u(t)=[B(E)](t) \\
& u(0)=u_{0} \quad, \quad \dot{u}(0)=u_{1}
\end{aligned}
$$


in the dual space $V^{*}$. This formulation illustrates the analogy between the infinite-dimensional, strongly damped elastic model and the familiar finite-dimensional relations (23).

\section{Model Well-Posedness}

As a prelude to establishing the well-posedness of the beam model with hysteretic $E$ - $P$ relations, we provide a lemma which quantifies the smoothness of the input operator.

Lemma 1 Consider field inputs $E \in C[0, T]$. The input operator $B$ defined by (33) then satisfies

$$
B(E) \in L^{2}\left(0, T ; V^{*}\right)
$$

Proof. In Appendix A, we establish that for continuous input fields $E$, the polarization satisfies $P \in C[0, T]$ which implies that $b$ defined by (35) satisfies $b(\cdot): C[0, T] \rightarrow C[0, T]$. Hence the norm

$$
\|[B(E)](t)\|_{V^{*}}=\sup _{v \in V} \frac{|[b(E)](t) \cdot g(v)|}{\|v\|_{V}}
$$

exists for each $t \in[0, T]$. Since $\|[B(E)](t)\|_{V^{*}}=|[b(E)](t)| \cdot\|g\|_{V^{*}}$, it follows that

$$
\|B(E)\|_{L^{2}\left(0, T ; V^{*}\right)}^{2} \leq \max _{t \in[0, T]}\left\{|[b(E)](t)|^{2}\right\} \cdot T \cdot\|g\|_{V^{*}}^{2}
$$

which implies that

$$
B(E) \in L^{2}\left(0, T ; V^{*}\right)
$$

The well-posedness of the model is established by the following theorem whose proof follows directly from Theorem 4.1 of [2] or Theorem 2.1 and Remark 2.1 of [1].

Theorem 2 Let $\sigma_{1}$ and $\sigma_{2}$ be given by (31) and consider continuous field inputs $E \in C[0, T]$. There then exists a unique solution $w$ to (36), or equivalently (38), which satisfies

$$
\begin{aligned}
& u \in C(0, T ; V) \\
& \dot{u} \in C(0, T ; X) .
\end{aligned}
$$

\subsection{Shell Model}

Similar well-posedness results can be obtained for the shell model (29) through consideration of an analogous Hilbert space formulation of the model. Details regarding the construction of appropriate inner product spaces, sesquilinear forms, and operators can be found in [25, 30].

\section{Numerical Approximation Techniques}

To implement the distributed models for either the rectangular stacked actuator or the cylindrical actuator, it is necessary to develop appropriate approximation techniques to discretize the modeling PDE. To accomplish this, we consider general Galerkin methods in which basis functions are comprised of spline or spline-Fourier tensor products. The resulting methods can accommodate a variety of boundary conditions, are sufficiently accurate to resolve fine-scale dynamics, and can be employed for constructing reduced-order POD approximates for real-time implementation. 


\subsection{Stacked Actuator Model}

To approximate the weak form of the stacked actuator model (20), we employ a finite element discretization and a finite difference discretization in time. The semidiscrete system resulting from the finite element approximation is appropriate for finite dimensional, continuous time control design whereas the fully discrete system is amenable to simulations and control implementation.

To obtain a semidiscrete system, we consider a uniform partition of $[0, \ell]$ with points $x_{j}=j h, j=$ $0,1, \cdots, N$ with step size $h=\ell / N$ where $N$ denotes the number of subintervals. The spatial basis $\left\{\phi_{j}\right\}_{j=1}^{N}$ is then comprised of linear splines

$$
\begin{aligned}
& \phi_{j}(x)=\frac{1}{h}\left\{\begin{array}{l}
\left(x-x_{j-1}\right), x_{j-1} \leq x<x_{j} \\
\left(x_{j+1}-x\right), x_{j} \leq x \leq x_{j+1} \\
0, \text { otherwise }
\end{array} \quad, \quad i=1, \cdots, N-1\right. \\
& \phi_{N}(x)=\frac{1}{h}\left\{\begin{array}{l}
\left(x-x_{N-1}\right), x_{N-1} \leq x \leq x_{N} \\
0, \text { otherwise }
\end{array}\right.
\end{aligned}
$$

(see [21] for details regarding the convergence analysis for the method). The solution $u(t, x)$ to $(20)$ is subsequently approximated by the expansion

$$
u^{N}(t, x)=\sum_{j=1}^{N} u_{j}(t) \phi_{j}(x)
$$

Through construction, the approximate solution satisfies the essential boundary condition $u^{N}(t, 0)=$ 0 and can attain arbitrary displacements at $x=\ell$.

The projection of the problem (20) onto the finite dimensional subspace $V^{N}$ yields the semidiscrete system

$$
\begin{aligned}
& \dot{\mathbf{z}}(t)=\mathbb{A} \mathbf{z}(t)+A\left[a_{1}\left(P(t)-P_{R}\right)+a_{2}\left(P(t)-P_{R}\right)^{2}\right] \mathbf{B} \\
& \mathbf{z}(0)=\mathbf{z}_{0}
\end{aligned}
$$

where $\mathbf{z}(t)=\left[u_{1}(t), \cdots, u_{N}(t), \dot{u}_{1}(t), \cdots, \dot{u}_{N}(t)\right]^{T}$ and

$$
\mathbb{A}=\left[\begin{array}{cc}
0 & \mathbb{I} \\
-\mathbb{M}^{-1} \mathbb{K} & -\mathbb{M}^{-1} \mathbb{Q}
\end{array}\right] \quad, \quad \mathbf{B}=\left[\begin{array}{c}
0 \\
\mathbb{M}^{-1} \mathbf{b}
\end{array}\right]
$$

The mass, stiffness and damping matrices have the components

$$
\begin{aligned}
& {[\mathbb{M}]_{i j}=\left\{\begin{array}{l}
\int_{0}^{\ell} \rho A \phi_{i} \phi_{j} d x \quad, \quad i \neq N \text { or } j \neq N \\
\int_{0}^{\ell} \rho A \phi_{i} \phi_{j} d x+m_{\ell}, \quad i=N \text { and } j=N
\end{array}\right.} \\
& {[\mathbb{K}]_{i j}=\left\{\begin{array}{l}
\int_{0}^{\ell} Y A \phi_{i}^{\prime} \phi_{j}^{\prime} d x \quad, \quad i \neq N \text { or } j \neq N \\
\int_{0}^{\ell} Y A \phi_{i}^{\prime} \phi_{j}^{\prime} d x+k_{\ell}, i=N \text { and } j=N
\end{array}\right.}
\end{aligned}
$$


and

$$
[\mathbb{Q}]_{i j}=\left\{\begin{array}{l}
\int_{0}^{\ell} c A \phi_{i}^{\prime} \phi_{j}^{\prime} d x \quad, \quad i \neq N \text { or } j \neq N \\
\int_{0}^{\ell} c A \phi_{i}^{\prime} \phi_{j}^{\prime} d x+c_{\ell} \quad, \quad i=N \text { and } j=N,
\end{array}\right.
$$

and the force vector is defined by

$$
[\mathbf{b}]_{i}=\int_{0}^{\ell} \phi_{i}^{\prime} d x
$$

The system (40) can be employed for finite-dimensional control design. For subsequent implementation, we consider a temporal discretization of (40) using a modified trapezoid rule. For temporal stepsizes $\Delta t$, this yields the difference equation

$$
\mathbf{z}_{k+1}=\mathbb{W} \mathbf{z}_{k}+\frac{1}{2}\left[a_{1} \widetilde{P}\left(t_{k}\right)+a_{1} \widetilde{P}\left(t_{k+1}\right)+a_{2} \widetilde{P}^{2}\left(t_{k}\right)+a_{2} \widetilde{P}^{2}\left(t_{k+1}\right)\right] \mathbb{V} \mathbf{B}
$$

where $\widetilde{P}=P-P_{R}, t_{j}=j \Delta t, \mathbf{z}_{j}$ approximates $\mathbf{z}\left(t_{j}\right)$, and

$$
\mathbb{W}=\left(\mathbb{I}-\frac{\Delta t}{2} \mathbb{A}\right)^{-1}\left(\mathbb{I}+\frac{\Delta t}{2} \mathbb{A}\right) \quad, \quad \mathbb{V}=\Delta t\left(\mathbb{I}-\frac{\Delta t}{2} \mathbb{A}\right)^{-1}
$$

This yields an A-stable method requiring moderate storage and providing moderate accuracy.

\subsection{Cylindrical Actuator Model}

Due to the inherent coupling between longitudinal, circumferential, and transverse displacements in combination with the 2-D support of the middle surface, the numerical approximation of the model for the cylindrical actuator is significantly more complicated than the approximation of the stacked actuator model. Among the issues which must be addressed when constructing finite element or general Galerkin methods for the shell is the choice of elements which avoid shear and membrane locking and the maintenance of boundary conditions. We summarize here a spline-based Galerkin method developed in [7] for thin shells and direct the reader to that source for details regarding the construction of constituent matrices and convergence properties of the method. Details regarding the use of this approximation method for LQR control of shells utilizing PZT actuators can be found in $[8]$.

The bases for the $u, v$ and $w$ displacements are respectively taken to be

$$
\Phi_{u_{k}}(\theta, x)=e^{i m \theta} \phi_{u_{n}}(x) \quad, \quad \Phi_{v_{k}}(\theta, x)=e^{i m \theta} \phi_{v_{n}}(x) \quad, \quad \Phi_{w_{k}}(\theta, x)=e^{i m \theta} \phi_{w_{n}}(x)
$$

where $\phi_{u_{n}}, \phi_{v_{n}}$ and $\phi_{w_{n}}$ are cubic $B$-splines modified to satisfy the boundary conditions (e.g., see page 79 of [21]). The approximating subspaces are

$$
V_{u}^{N}=\operatorname{span}\left\{\Phi_{u_{k}}\right\}_{k=1}^{N_{u}} \quad, \quad V_{v}^{N}=\operatorname{span}\left\{\Phi_{v_{k}}\right\}_{k=1}^{N_{v}} \quad, \quad V_{w}^{N}=\operatorname{span}\left\{\Phi_{w_{k}}\right\}_{k=1}^{N_{w}}
$$

and the approximate displacements are represented by the expansions

$$
\begin{aligned}
& u^{N}(t, \theta, x)=\sum_{k=1}^{N_{u}} u_{k}(t) \Phi_{u_{k}}(\theta, x) \\
& v^{N}(t, \theta, x)=\sum_{k=1}^{N_{v}} v_{k}(t) \Phi_{v_{k}}(\theta, x) \\
& w^{N}(t, \theta, x)=\sum_{k=1}^{N_{w}} w_{k}(t) \Phi_{w_{k}}(\theta, x) .
\end{aligned}
$$


The restriction of the problem (29) to the approximating subspaces and construction of the forcing vectors subsequently yields the matrix system

$$
\begin{aligned}
& \dot{\mathbf{z}}^{N}(t)=\mathbb{A} \mathbf{z}(t)+\left[a_{1}\left(P(t)-P_{R}\right)+a_{2}\left(P(t)-P_{R}\right)^{2}\right] \mathbf{B} \\
& \mathbf{z}(0)=\mathbf{z}_{0},
\end{aligned}
$$

where $\mathbf{z}=[\boldsymbol{\vartheta}(t), \dot{\boldsymbol{\vartheta}}(t)]^{T}$, with $\boldsymbol{\vartheta}(t)=[\mathbf{u}(t), \mathbf{v}(t), \mathbf{w}(t)]^{T}$, and

$$
\mathbb{A}=\left[\begin{array}{cc}
0 & \mathbb{I} \\
-\mathbb{M}^{-1} \mathbb{K} & -\mathbb{M}^{-1} \mathbb{Q}
\end{array}\right] \quad, \quad \mathbf{B}=\left[\begin{array}{c}
0 \\
\mathbb{M}^{-1} \mathbf{b}
\end{array}\right] .
$$

The reader is referred to $[7,25]$ for details concerning the construction of the mass, stiffness and damping matrices $\mathbb{M}, \mathbb{K}$ and $\mathbb{Q}$.

\section{Model Validation}

\section{Characterization of the Stacked Actuator}

We consider the capability of the modeling framework to characterize the dynamics of the stacked actuator depicted in Figure 2(a). The PZT actuator had a length of $\ell=2 \times 10^{-2} \mathrm{~m}$ and a square cross-sectional face of width $w=5 \times 10^{-3} \mathrm{~m}$ so that the cross-sectional area is $A=2.5 \times 10^{-5} \mathrm{~m}^{2}$. As illustrated in Figure 7, one end of the actuator was considered fixed whereas the other encountered elastic, damping and inertial effects due to the attached components of the stage mechanism.

To validate and illustrate properties of the models, we consider three regimes: (i) end displacements quantified by the lumped model (21) with the thermally inactive kernel (7) employed in the polarization model (15), (ii) displacements characterized by the lumped model with the thermally active polarization kernel (9), and (iii) end displacements quantified by the discretization (42) of the distributed model (20). It is illustrated that whereas the latter choice incorporates the distributed rod nature of the device, the fact that fields and stresses are uniform along the rod length implies that relative displacements are also uniform. A comparison of the ODE and PDE model predictions at the rod tip $(x=\ell)$ illustrates that as a result, the ODE provides a highly accurate characterization with significantly less computation cost. Hence the ODE model is advantageous for real-time experimental implementation.

The construction of the models requires the estimation of elastic, damping and electromechanical parameters in addition to identification of the densities $\nu_{1}$ and $\nu_{2}$. The densities were estimated through least squares fits to the data using the techniques detailed in [25, 27]. The manufacturer specifications $\rho=7600 \mathrm{~kg} / \mathrm{m}^{3}$ and $Y=7 \times 10^{10} \mathrm{~N} / \mathrm{m}^{2}$ were employed for the density and Young's modulus and remaining parameters were estimated through a least squares fit to the data. The resulting values are summarized in Table 1 . The relation between the rod and spring parameters is provided by (22).

\section{Lumped Model - No Thermal Activation in Polarization Relation}

We consider first the characterization of the biased minor loop data shown in Figure 3 and frequency-dependent data from Figure 4 using the lumped model (21) with the thermally inactive kernel (7) employed in the polarization model (15). It should be noted that the stage was disassembled between the quasistatic, biased minor loop experiments and the frequency-dependent experiments which necessitated the re-identification of densities for the two cases. 


\begin{tabular}{|l||cccccccc|}
\hline \multicolumn{10}{|c|}{ Distributed Model } \\
\hline Parameter & $\rho$ & $Y$ & $C$ & $m_{\ell}$ & $k_{\ell}$ & $c_{\ell}$ & $a_{1}$ & $a_{2}$ \\
Value & 7600 & $7 \times 10^{10}$ & $5 \times 10^{6}$ & 4.015 & $8.49 \times 10^{-5}$ & 440 & $1.54 \times 10^{11}$ & 0 \\
\hline \hline \multicolumn{7}{|c|}{ Lumped Model } \\
\hline Parameter & $m$ & $k$ & $c$ & $\widetilde{a}_{1}$ & $\widetilde{a}_{2}$ & & \\
Value & 4.21 & $8.75 \times 10^{7}$ & $1.52 \times 10^{5}$ & $8.75 \times 10^{7}$ & 0 & \\
\hline
\end{tabular}

Table 1: Parameters employed in the distributed (PDE) model (20) and lumped (ODE) model (21) for the stacked actuator.

In the first set of experiments, displacement data measured with an LVDT was collected at a sample rate of $0.1 \mathrm{~Hz}$ and four input field levels to generate a set of biased and nested transducer responses ranging from nearly linear to hysteretic and nonlinear as shown in Figures 3 and 9. The densities $\nu_{1}$ and $\nu_{2}$ and parameters summarized in Table 1 were obtained through a least squares fit to the full data set comprised of four loops. The resulting model accurately quantifies both the nest behavior and the hysteresis measured at increasing input levels.

In a second set of experiments, data was collected a frequencies ranging from $0.279 \mathrm{~Hz}$ to $27.9 \mathrm{~Hz}$ yielding the behavior shown in Figure 4. The data from four frequencies was used to re-identify
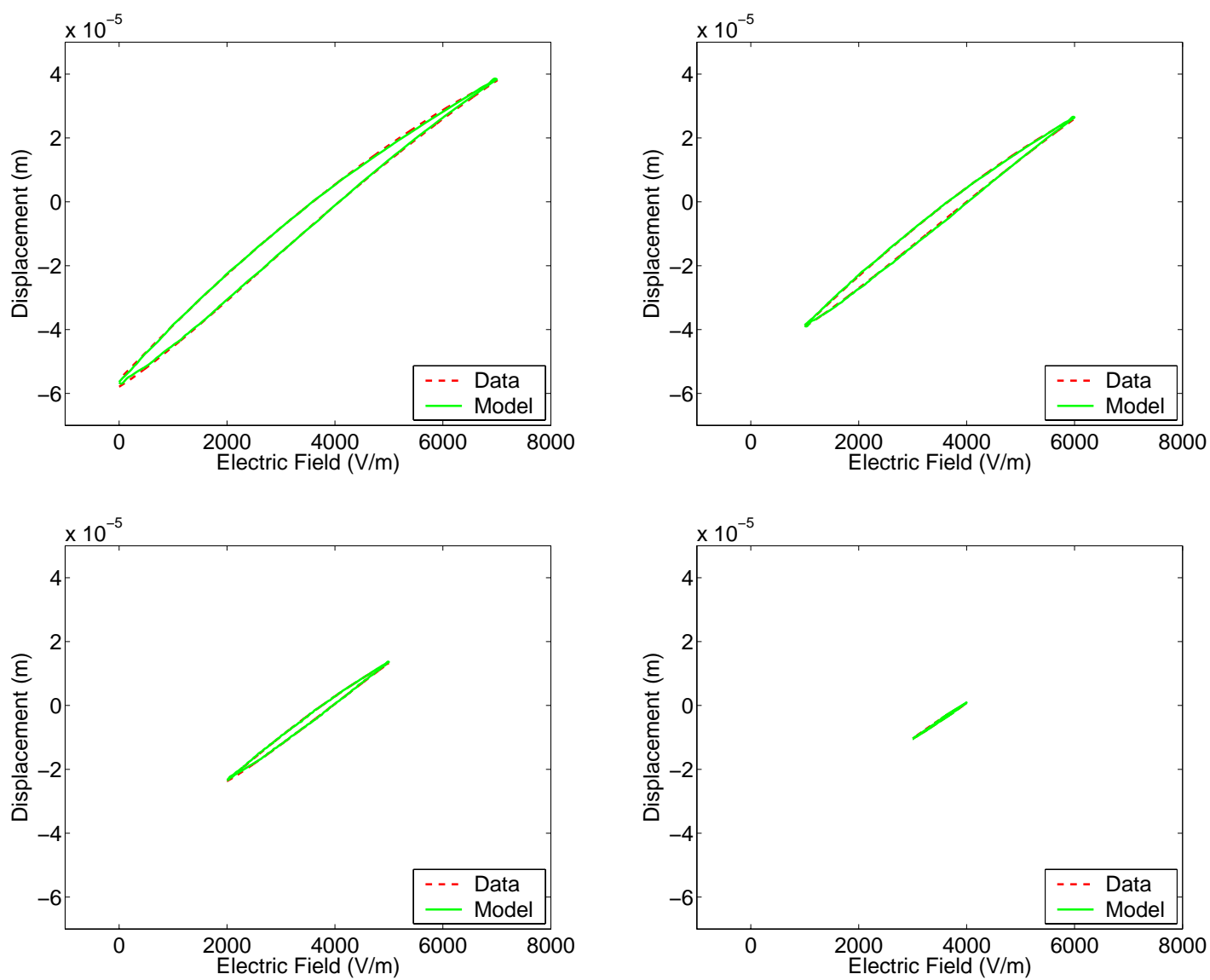

Figure 9: Characterization of AFM field-displacement behavior at $0.1 \mathrm{~Hz}$ using the ODE model (21) with the thermally inactive kernel (7). 
parameters in the polarization model, since the stage had been modified, thus yielding the fits shown in Figure 10. It is observed that the model characterizes the augmented hysteresis arising at higher frequencies but slightly overpredicts the increase in displacement following field reversal which is due primarily to inertial effects.

\section{Lumped Model - Thermal Activation in Polarization Relation}

We next employ the thermally active kernel (9) in the polarization model to incorporate relaxation effects. Parameters in the polarization model were again identified through a least squares fit to the four frequency data sets thus yielding the model fit shown in Figure 11. It is observed that use of this more general kernel provides additional accuracy at higher frequencies. Whereas this improves characterization capabilities, the added accuracy comes at the cost of decreased efficiency, and the criteria of accuracy versus efficiency must be balanced when employing the model for real-time control design as discussed in [12].

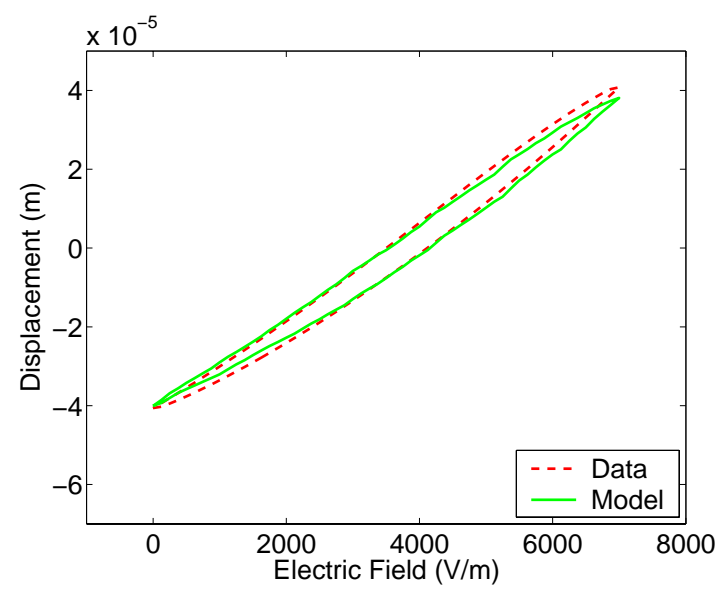

(a)

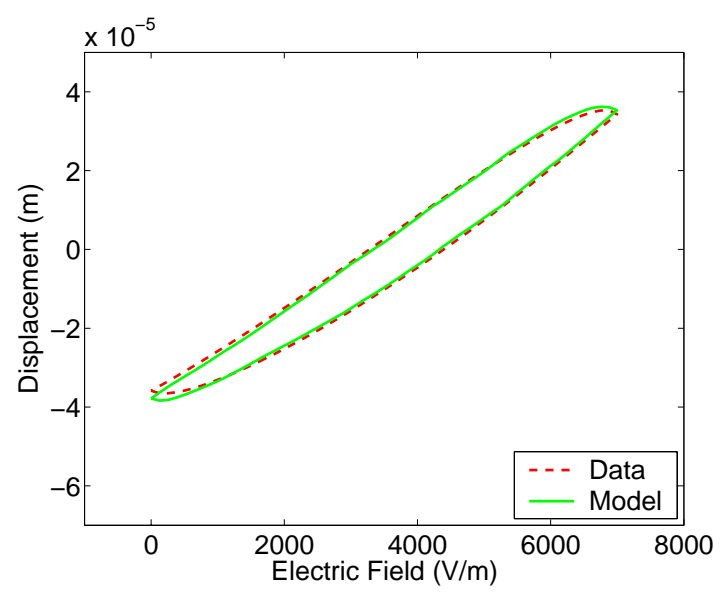

(c)

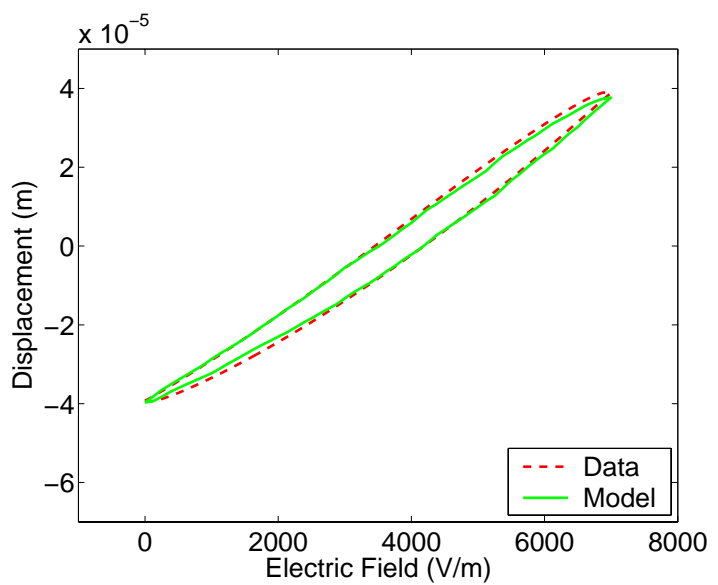

(b)

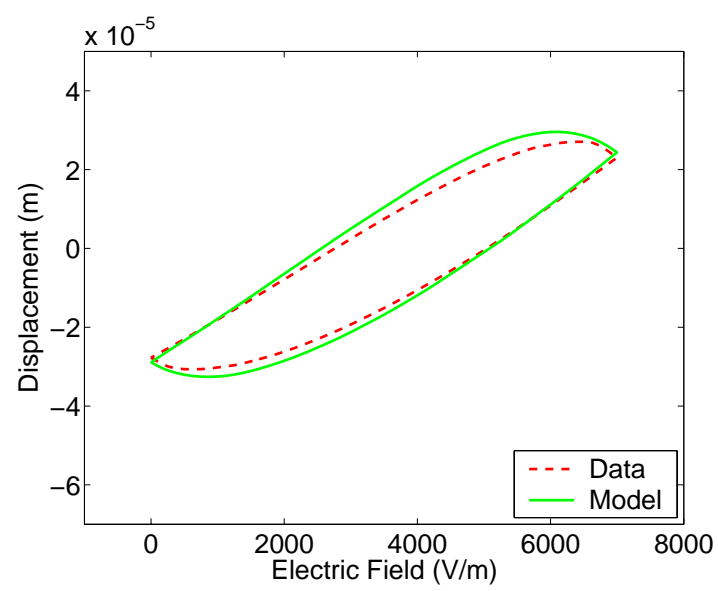

(d)

Figure 10: Characterization of AFM field-displacement behavior using the ODE model (21) with the thermally inactive kernel (7) with sample rates of (a) $0.279 \mathrm{~Hz}$, (b) $1.12 \mathrm{~Hz}$, c) $5.58 \mathrm{~Hz}$, and (d) $27.9 \mathrm{~Hz}$. 


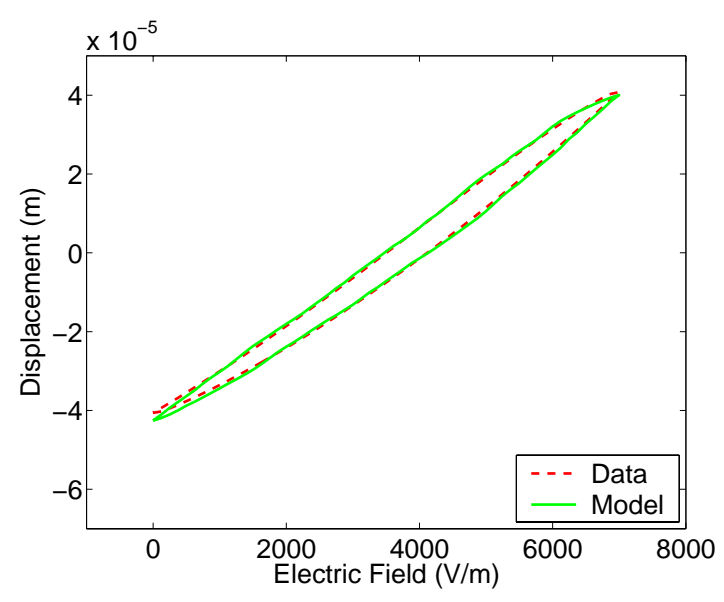

(a)

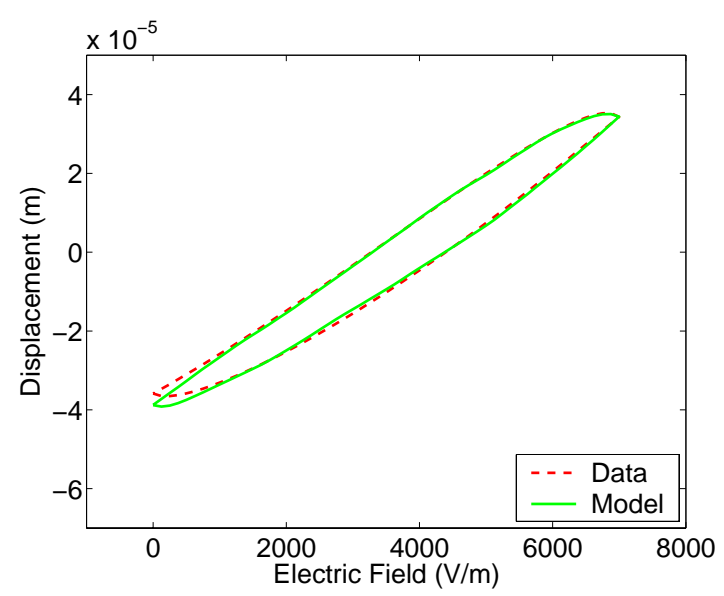

(c)

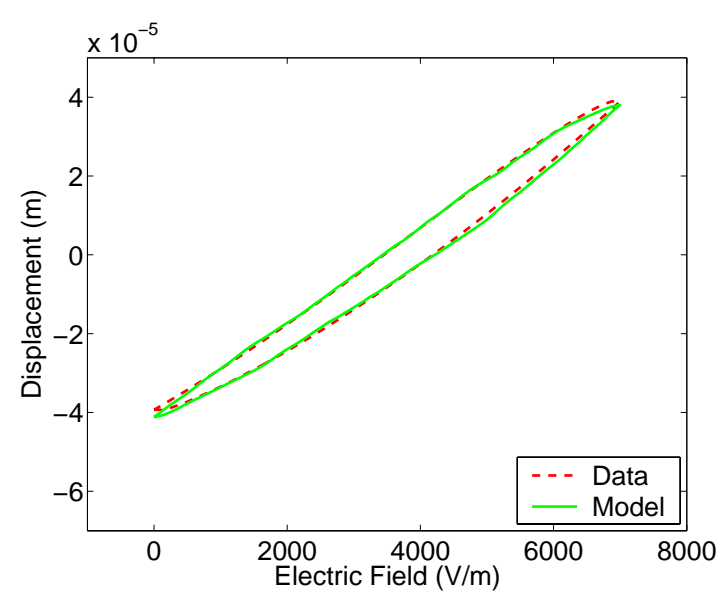

(b)

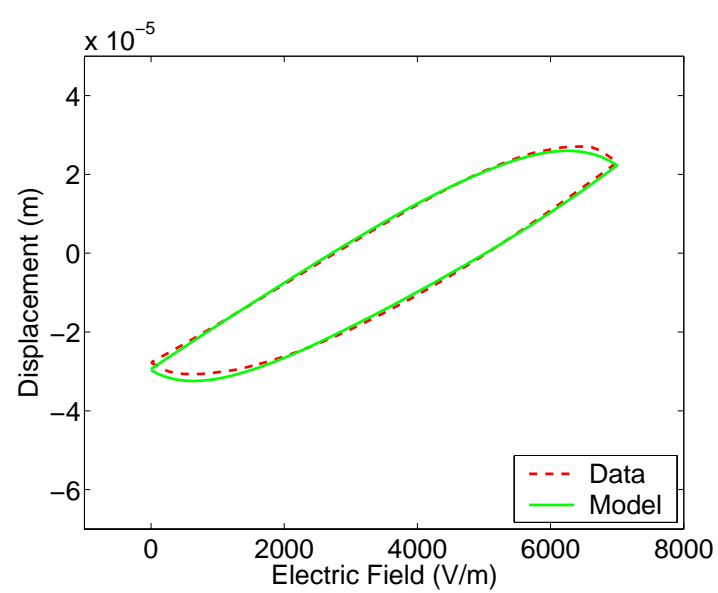

(d)

Figure 11: Characterization of AFM field-displacement behavior using the ODE model (21) with the thermally active kernel (9) with sample rates of (a) $0.279 \mathrm{~Hz}$, (b) $1.12 \mathrm{~Hz}$, c) $5.58 \mathrm{~Hz}$, and (d) $27.9 \mathrm{~Hz}$.

\section{Lumped Versus Distributed Models}

It has been observed that whereas quantification of the physics of the stacked actuator leads to the rod model (20), the fact that stresses and fields are uniform along the rod length implies that relative displacements will also be uniform. This motivates consideration of the lumped model (21) which yielded the fits shown in Figures 10 and 11.

To illustrate the validity of this assumption, the difference between the displacement $u(t, \ell)$, given by the discretization (42) of (20), and the displacement $u_{\ell}(t)$ resulting from (21) is plotted in Figure 12. We emphasize that when constructing the PDE model, we employed the parameter values summarized in Table 1 which are consistent with the spring parameters due to the relation (22). The maximal difference of $5 \times 10^{-10}$ is 5 orders of magnitude less than the micron-level displacements being characterized thus verifying the validity of the ODE model in this regime. The accuracy of the ODE model has important ramifications for control design since the discretized ODE model is significantly more efficient to implement than the discretized PDE model. 


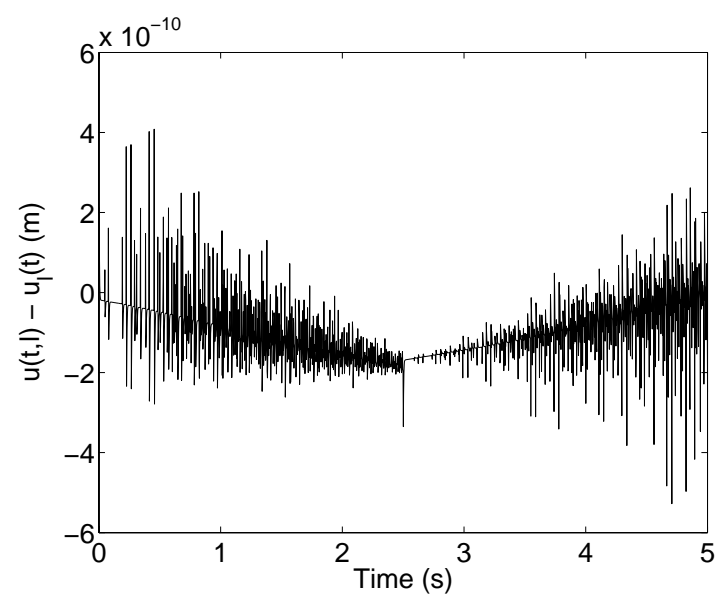

Figure 12: Difference between the displacement $u(t, \ell)$ given by the distributed model $(20)$ and $u_{\ell}(t)$ given by the lumped model (21).

\section{Characterization of the Stacked Actuator}

We discuss here the performance of the cylindrical shell model detailed in Section 3.2, when discretized using the Galerkin techniques summarized in Section 5.2, for characterizing the longitudinal displacements of the cylindrical PZT shell transducer depicted in Figures 2(b) and 7 . Whereas the cylindrical PZT elements employed in this design are more complex than the rod elements used in the stage design depicted in Figure 2(a), the overall transducer is simpler and has the advantage of enhanced vibration isolation and diminished hysteresis.

The experimental cylindrical transducer had a length of $\ell=0.0396 \mathrm{~m}$, radius of $R=0.0056 \mathrm{~m}$ and thickness $h=0.0015 \mathrm{~m}$. The manufacturer specifications $\rho=7600 \mathrm{~kg} / \mathrm{m}^{3}$ and $Y=7.1 \times 10^{1} 0$ $\mathrm{N} / \mathrm{m}^{2}$ were employed for the density and Young's modulus, and remaining model parameters were estimated through a least squares fit to the data.

The longitudinal displacement $u^{N}$ provided by (43) is compared with experimental data in Fig-

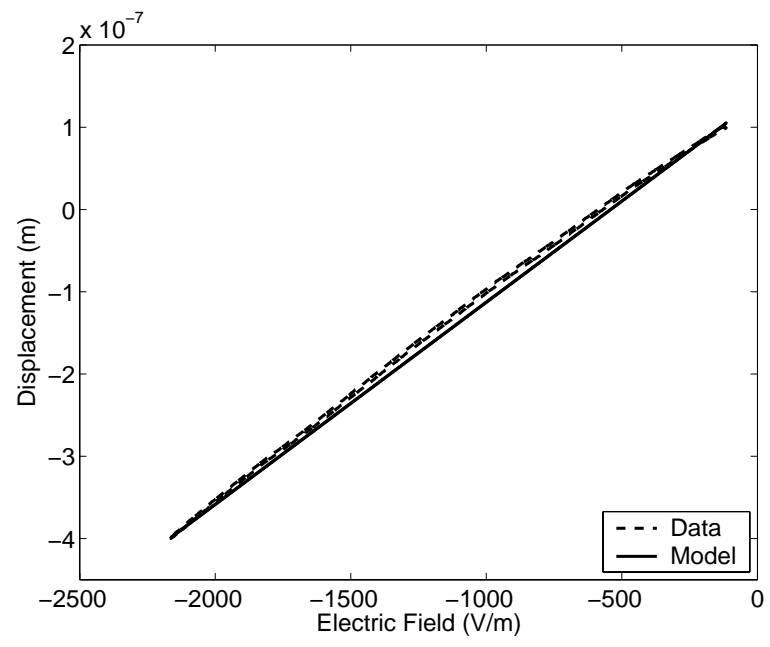

Figure 13: Characterization of the relation between the field and longitudinal displacements for the cylindrical actuator depicted in Figures 2(b) and 7. 
ure 13. We note that due to the inherent coupling between the longitudinal, circumferential and transverse displacements, $u, v$ and $w$ in the model (24), the approximate displacements (43) are also coupled and all are obtained through solution of (44) - we plot only $u^{N}$ since it corresponds to measured data. The nearly linear behavior of both the data and model response illustrates an advantage of this design and the property that the hysteretic $E$ - $P$ model (12) yields approximately linear behavior in low drive regimes. The fidelity of the model further illustrates the accuracy and flexibility of the modeling framework.

\section{Concluding Remarks}

The characterization framework developed here quantifies both the approximately linear and hysteretic properties of PZT device employed in atomic force microscope (AFM) positioning mechanisms. In the first step of the development, constitutive relations are constructed through a combination of energy analysis at the lattice level and stochastic homogenization techniques based on the assumption that certain parameters are manifestations of underlying distributions. These relations quantify the frequency-dependent hysteresis exhibited by the materials for general drive regimes while reducing to approximately linear behavior at low drive regimes. In the second step of the of the development, these constitutive relations are used to construct lumped and distributed rod and shell models for the various PZT transducer geometries. The accuracy of the models is illustrated through comparison with experimental data from AFM stages.

An important property of the framework is the fact that resulting models can be approximately inverted with nearly the same efficiency as the forward models [12]. This provides the framework with the capability for providing inverse compensators for linear control design $[18,19]$. The implementation of feedback control designs for high speed scanning, using these model-based compensators, is under present investigation.

\section{A Continuity of the Polarization Model}

We establish here the continuity of the homogenized energy model (12),

$$
[P(E)](t)=\int_{0}^{\infty} \int_{-\infty}^{\infty}\left[\bar{P}\left(E+E_{I} ; E_{c}, \xi\right)\right](t) \nu_{1}\left(E_{c}\right) \nu_{2}\left(E_{I}\right) d E_{I} d E_{c}
$$

as a function of both field and time in the case of negligible thermal activation. The densities $\nu_{1}$ and $\nu_{2}$ satisfy the conditions (13) and the kernel $\bar{P}$ has the form

$$
\bar{P}(E)=\frac{E}{\eta}+P_{R} \delta\left(E ; E_{c}, E_{I}\right)
$$

specified in (7).

We first note that there are at most three values at which $\delta$ can change sign: $-E_{c}, E_{c}$ and $-E_{c} \leq E_{T} \leq E_{c}$. The third is determined by the initial dipole distribution $\xi$, as depicted in Figure $14(\mathrm{a})$, and is typically chosen so that $E_{T}=0$ when $E+E_{I}=0$. 


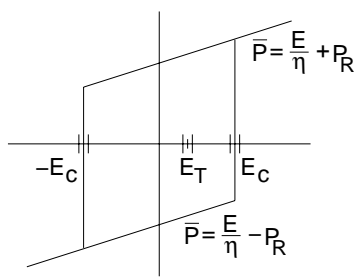

(a)

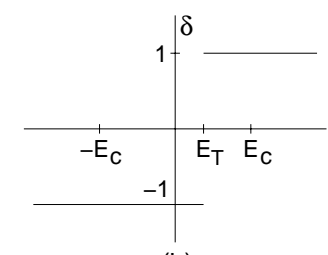

(b)

Figure 14: (a) Points $E+E_{I}=-E_{c}, E_{c}$ and $E_{T}$ at which $\delta= \pm 1$ changes sign, and (b) behavior of $\delta$ associated with the initial dipole distribution at $E+E_{I}=E_{T}$.

We also note that the decay conditions (13) dictate that $\nu_{1}$ and $\nu_{2}$ satisfy the relations

$$
\begin{aligned}
& \left|\nu_{2}\left(E_{I}\right)\right| \leq c_{2} \\
& \int_{-\infty}^{\infty} \nu_{2}\left(E_{I}\right) d E_{I} \leq b_{2} \\
& \int_{0}^{\infty} \nu_{1}\left(E_{c}\right) d E_{c} \leq b_{1}
\end{aligned}
$$

where $b_{1}, b_{2}$ and $c_{2}$ are finite constants.

To establish the continuity of $P$ with respect to $E$, we consider the behavior at field values $E_{0}$ and $E_{1}$ where, without loss of generality, we take $E_{0}<E_{1}$. When integrating with respect to $E_{I}$, we decompose the interval $(-\infty, \infty)$ into seven regions delineated by the points $-E_{c}, E_{c}, E_{T}$ as shown in Figure 14(a). For this decomposition, we note that

$$
\left|\bar{P}\left(E_{1}+E_{I} ; E_{c}, \xi\right)-\bar{P}\left(E_{0}+E_{I} ; E_{c}, \xi\right)\right|=\left\{\begin{array}{c}
\frac{1}{\eta}\left(E_{1}-E_{0}\right), \\
-E_{c}, E_{c}, E_{T} \\
\frac{1}{\eta}\left(E_{1}-E_{0}\right)+2 P_{R}, \text { region includes } \\
-E_{c}, E_{c}, E_{T}
\end{array}\right.
$$

To consolidate notation, we define the integrals

$$
\begin{aligned}
& I(a, b)=\int_{a}^{b} \frac{1}{\eta}\left(E_{1}-E_{0}\right) \nu_{2}\left(E_{I}\right) d E_{I} \\
& I_{P_{R}}(a, b)=\int_{a}^{b}\left[\frac{1}{\eta}\left(E_{1}-E_{0}\right)+2 P_{R}\right] \nu_{2}\left(E_{I}\right) d E_{I} .
\end{aligned}
$$


It subsequently follows that

$$
\begin{aligned}
&\left|P\left(E_{1}\right)-P\left(E_{0}\right)\right| \leq \int_{0}^{\infty}\left\{\left|I\left(-\infty,-E_{c}-E_{1}\right)\right|+\left|I_{P_{R}}\left(-E_{c}-E_{1},-E_{c}-E_{0}\right)\right|\right. \\
&+\left|I\left(-E_{c}-E_{0}, E_{T}-E_{1}\right)\right|+\left|I_{P_{R}}\left(E_{T}-E_{1}, E_{T}-E_{0}\right)\right| \\
&+\left|I\left(E_{T}-E_{0}, E_{c}-E_{1}\right)\right|+\left|I_{P_{R}}\left(E_{c}-E_{1}, E_{c}-E_{0}\right)\right| \\
&\left.+\left|I\left(E_{c}-E_{0}, \infty\right)\right|\right\} \nu_{1}\left(E_{c}\right) d E_{c} \\
& \leq\left(E_{1}-E_{0}\right) \int_{0}^{\infty}\left\{\frac{4 c_{2}}{\eta}+3 b_{2}\left[\frac{1}{\eta}\left(E_{1}-E_{0}\right)+2 P_{R}\right]\right\} \nu_{1}\left(E_{c}\right) d E_{c} \\
& \leq\left(E_{1}-E_{0}\right) b_{1}\left(\frac{4 c_{2}}{\eta}+3 b_{2}\left[\frac{1}{\eta}\left(E_{1}-E_{0}\right)+2 P_{R}\right]\right) .
\end{aligned}
$$

For $\varepsilon>0$, take

$$
\delta=\min \left\{\frac{\varepsilon}{b_{1}\left(\frac{4 c_{2}}{\eta}+3 b_{2}\left[\frac{1}{\eta}\left(E_{1}-E_{0}\right)+2 P_{R}\right]\right)}, 1\right\} .
$$

Under the assumption that $E$ is continuous in time and $E_{0}=E\left(t_{0}\right), E_{1}=E\left(t_{1}\right)$, for every $\delta>0$ there exists $\widetilde{\delta}>0$ such that if $\left|t_{1}-t_{0}\right|<\widetilde{\delta}$, we are guaranteed that $\left|E_{1}-E_{0}\right|<\delta$. It follows that if $\left|t_{1}-t_{0}\right|<\widetilde{\delta}$, the polarization values satisfy the bound

$$
\left|[P(E)]\left(t_{1}\right)-[P(E)]\left(t_{0}\right)\right| \leq \varepsilon
$$

thus establishing the continuity of the hysteresis model. This holds for all major and minor loops. As illustrated in Figure 6, the behavior of the model which incorporates thermal activation is smoother than the thermally inactive case considered here. For brevity, we omit the proof of this second case.

\section{Acknowledgements}

The research of A.H. was supported by the DARPA subcontract 1000-G-CF980 and the first four authors were supported by the NSF grant CMS-0201560. Additionally, the research of R.C.S. was supported in part through the NSF grant CMS-0099764 and in part by the Air Force Office of Scientific Research through the grants AFOSR-F49620-01-1-0107 and AFOSR-FA9550-04-1-0203.

\section{References}

Note: Center for Research in Scientific Computation Technical Reports can be accessed at the web site http://www.ncsu.edu/crsc/reports.html.

[1] H.T. Banks, K. Ito and Y. Wang, "Well-posedness for damped second order systems with unbounded input operators," Differential and Integral Equations, 8, pp. 587-606, 1995.

[2] H.T. Banks, R.C. Smith and Y. Wang, Smart Material Structures: Modeling, Estimation and Control, Masson/John Wiley, Paris/Chichester, 1996. 
[3] W. Chen and C.S. Lynch, "A model for simulating polarization switching and AF-F phase changes in ferroelectric ceramics," Journal of Intelligent Material Systems and Structures, 9, pp. 427-431, 1998.

[4] W. Chen and C.S. Lynch, "A micro-electro-mechanical model for polarization switching of ferroelectric materials," Acta Materialia, 46(15), pp. 5303-5311, 1998.

[5] D. Croft, G. Shed and S. Devasia, "Creep, hysteresis, and vibration compensation for piezoactuators: Atomic force microscopy application," Journal of Dynamic Systems, Measurement, and Control, 23, pp. 35-43, 2001.

[6] A. Daniele, S. Salapaka, M.V. Salapaka and M. Dahleh, "Piezoelectric scanners for atomic force microscopes: Design of lateral sensors, identification and control," Proceedings of the America Control Conference, San Diego, CA, pp. 253-257, 1999.

[7] R.C.H. del Rosario and R.C. Smith, "Spline approximation of thin shell dynamics," International Journal for Numerical Methods in Engineering, 40, pp. 2807-2840, 1997.

[8] R.C.H. del Rosario and R.C. Smith, "LQR control of thin shell dynamics: formulation and numerical implementation," Journal of Intelligent Material Systems and Structures, 9(4), pp. 301$320,1998$.

[9] P. Ge and M. Jouaneh, "Modeling hysteresis in piezoceramic actuators," Precision Engineering, 17, pp. 211-221, 1995.

[10] P.K. Hansma, V.B. Elings, O. Marti and C.E. Bracker, "Scanning tunneling microscopy and atomic force microscopy: Application to biology and technology," Science, 242, pp. 209-242, 1988.

[11] A.G. Hatch, Model Development and Control Design for Atomic Force Microscopy, PhD Dissertation, North Carolina State University, Raleigh, NC, 2004.

[12] A.G. Hatch, R.C. Smith, T. De and M.V. Salapaka, "Construction and experimental implementation of a model-based inverse filter to attenuate hysteresis in ferroelectric transducers," CRSC Technical Report CRSC-TR05-06; IEEE Transactions on Control Systems Technology, submitted.

[13] L. Huang and H.F. Tiersten, "An analytic description of slow hysteresis in polarized ferroelectric ceramic actuators," Journal of Intelligent Material Systems and Structures, 9, pp. 417-426, 1998.

[14] C.M. Landis, "Non-linear constitutive modeling of ferroelectrics," Current Opinion in Solid State and Materials Science, 8, pp. 59-69, 2004.

[15] W. Lu, D.-N. Fang and K-C. Hwang, "Nonlinear electric-mechanical behavior and micromechanics modelling of ferroelectric domain evolution," Acta Materialia, 47(10), pp. 2913-2926, 1999.

[16] J.A. Main, E. Garcia and D.V. Newton, "Precision position control of piezoelectric actuators using charge feedback," Journal of Guidance, Control, and Dynamics. 18(5), pp. 1068-73, 1995.

[17] J.A. Main, D. Newton, L. Massengil and E. Garcia, "Efficient power amplifiers for piezoelectric applications," Smart Materials and Structures, 5(6), pp. 766-775, 1996. 
[18] J.M. Nealis and R.C. Smith, "H्H $\infty_{\infty}$ Control Design for a Magnetostrictive Transducer," Proc. 42nd IEEE Conference on Decision and Control, Maui, HA, pp. 1801-1806, 2003.

[19] J.M. Nealis and R.C. Smith, "Model-Based Robust Control Design for Magnetostrictive Transducers Operating in Hysteretic and Nonlinear Regimes," CRSC Technical Report CRSC-TR0325; IEEE Transactions on Control Systems Technology, submitted.

[20] M.B. Ozer and T.J. Royston, "Modeling the effect of piezoceramic hysteresis in structural vibration control," Smart Structures and Materials 2001, Proceedings of the SPIE, Volume 4326, pp. 89-100, 2001.

[21] P.M. Prenter, Splines and Variational Methods, Wiley, New York, 1975.

[22] G. Robert, D. Damjanovic and N. Setter, "Preisach modeling of piezoelectric nonlinearity in ferroelectric ceramics," Journal of Applied Physics, 89(9), pp. 5067-5074, 2001.

[23] D. Rugar, O. Züger, S.T. Hoen, C.S. Yannoni, H.-M. Vieth and R.D. Kendrick, "Force detection of nuclear magnetic resonance," Science, 264, pp. 1560-1563, 1994.

[24] S. Salapaka, A. Sebastian, J.P. Cleveland and M.V. Salapaka, "High bandwidth nano-positioner: A robust control approach," Review of Scientific Instruments, 73(9), pp. 3232-3241, 2002.

[25] R.C. Smith, Smart Material Systems: Model Development, SIAM, Philadelphia, PA, 2005.

[26] R.C. Smith and A. Hatch, "Parameter estimation techniques for nonlinear hysteresis models," Smart Structures and Materials 2004, Proceedings of the SPIE, Volume 5383, pp. 155-163, 2004.

[27] R.C. Smith, A. Hatch, B. Mukherjee and S. Liu, "A homogenized energy model for hysteresis in ferroelectric materials: General density formulation," CRSC Technical Report CRSC-TR04-23; Journal of Intelligent Material Systems and Structures, to appear.

[28] R.C. Smith and C.L. Hom, "Domain wall theory for ferroelectric hysteresis," Journal of Intelligent Material Systems and Structures, 10(3), pp. 195-213, 1999.

[29] R.C. Smith and Z. Ounaies, "A domain wall model for hysteresis in piezoelectric materials," Journal of Intelligent Material Systems and Structures, 11(1), pp. 62-79, 2000.

[30] R.C. Smith and M. Salapaka, "Model development for the positioning mechanisms in an atomic force microscope," International Series of Numerical Mathematics, Vol 143, pp. 249-269, 2002.

[31] R.C. Smith, S. Seelecke, M.J. Dapino and Z. Ounaies, "A unified model for hysteresis in ferroic materials," Smart Structures and Materials 2003, Proceedings of the SPIE, Volume 5049, pp. 8899, 2003.

[32] R.C. Smith, S. Seelecke, M.J. Dapino and Z. Ounaies, "A unified framework for modeling hysteresis in ferroic materials," Journal of the Mechanics and Physics of Solids, submitted.

[33] R.C. Smith, S. Seelecke, Z. Ounaies and J. Smith, "A free energy model for hysteresis in ferroelectric materials," Journal of Intelligent Material Systems and Structures, 14(11), pp. 719-739, 2003.

[34] S.A. Wolf, D.D. Awschalom, R.A. Buhrman, J.M. Daughton, S. von Molnár, M.L. Chtchelkanova and D.M. Teger, "Spintronics: A spin-based electronics vision for the future," Science, 294, pp. 1488-1495, 2001. 